University of Nebraska - Lincoln

DigitalCommons@University of Nebraska - Lincoln

\title{
Synthesis of Colloidal Mn2+:ZnO Quantum Dots and High-TC Ferromagnetic Nanocrystalline Thin Films
}

\author{
Nick S. Norberg \\ University of Washington \\ Kevin R. Kittilstved \\ University of Washington \\ James E. Amonette \\ Pacific Northwest National Laboratory \\ Ravi K. Kukkadapu \\ Pacific Northwest National Laboratory, ravi.kukkadapu@pnl.gov \\ Dana A. Schwartz \\ University of Washington \\ See next page for additional authors
}

Follow this and additional works at: https://digitalcommons.unl.edu/usdoepub

Part of the Bioresource and Agricultural Engineering Commons

Norberg, Nick S.; Kittilstved, Kevin R.; Amonette, James E.; Kukkadapu, Ravi K.; Schwartz, Dana A.; and Gamelin, Daniel R., "Synthesis of Colloidal Mn2+:ZnO Quantum Dots and High-TC Ferromagnetic Nanocrystalline Thin Films" (2004). US Department of Energy Publications. 167.

https://digitalcommons.unl.edu/usdoepub/167

This Article is brought to you for free and open access by the U.S. Department of Energy at DigitalCommons@University of Nebraska - Lincoln. It has been accepted for inclusion in US Department of Energy Publications by an authorized administrator of DigitalCommons@University of Nebraska - Lincoln. 


\section{Authors}

Nick S. Norberg, Kevin R. Kittilstved, James E. Amonette, Ravi K. Kukkadapu, Dana A. Schwartz, and Daniel R. Gamelin 


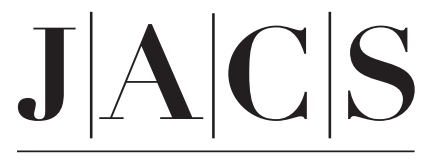

A R T I C L E S

Published on Web 07/14/2004

\title{
Synthesis of Colloidal $\mathrm{Mn}^{2+}: \mathrm{ZnO}$ Quantum Dots and High- $T_{\mathrm{C}}$ Ferromagnetic Nanocrystalline Thin Films
}

\author{
Nick S. Norberg, ${ }^{\dagger}$ Kevin R. Kittilstved,${ }^{\dagger}$ James E. Amonette, ${ }^{\ddagger}$ Ravi K. Kukkadapu, ${ }^{\ddagger}$ \\ Dana A. Schwartz, ${ }^{\dagger}$ and Daniel R. Gamelin*, ${ }^{\star}$ \\ Contribution from the Department of Chemistry, University of Washington, \\ Seattle, Washington 98195-1700, and Pacific Northwest National Laboratory, \\ Richland, Washington 99352
}

Received March 18, 2004; E-mail: Gamelin@chem.washington.edu

\begin{abstract}
We report the synthesis of colloidal $\mathrm{Mn}^{2+}$-doped $\mathrm{ZnO}\left(\mathrm{Mn}^{2+}: \mathrm{ZnO}\right)$ quantum dots and the preparation of room-temperature ferromagnetic nanocrystalline thin films. $\mathrm{Mn}^{2+}: \mathrm{ZnO}$ nanocrystals were prepared by a hydrolysis and condensation reaction in DMSO under atmospheric conditions. Synthesis was monitored by electronic absorption and electron paramagnetic resonance (EPR) spectroscopies. $\mathrm{Zn}(\mathrm{OAc})_{2}$ was found to strongly inhibit oxidation of $\mathrm{Mn}^{2+}$ by $\mathrm{O}_{2}$, allowing the synthesis of $\mathrm{Mn}^{2+}: \mathrm{ZnO}$ to be performed aerobically. $\mathrm{Mn}^{2+}$ ions were removed from the surfaces of as-prepared nanocrystals using dodecylamine to yield high-quality internally doped $\mathrm{Mn}^{2+}: \mathrm{ZnO}$ colloids of nearly spherical shape and uniform diameter $(6.1 \pm 0.7 \mathrm{~nm})$. Simulations of the highly resolved X-and Q-band nanocrystal EPR spectra, combined with quantitative analysis of magnetic susceptibilities, confirmed that the manganese is substitutionally incorporated into the $\mathrm{ZnO}$ nanocrystals as $\mathrm{Mn}^{2+}$ with very homogeneous speciation, differing from bulk $\mathrm{Mn}^{2+}: \mathrm{ZnO}$ only in the magnitude of $D$-strain. Robust ferromagnetism was observed in spincoated thin films of the nanocrystals, with $300 \mathrm{~K}$ saturation moments as large as $1.35 \mu_{\mathrm{B}} / \mathrm{Mn}^{2+}$ and $T_{\mathrm{C}}>$ $350 \mathrm{~K}$. A distinct ferromagnetic resonance signal was observed in the EPR spectra of the ferromagnetic films. The occurrence of ferromagnetism in $\mathrm{Mn}^{2+}: \mathrm{ZnO}$ and its dependence on synthetic variables are discussed in the context of these and previous theoretical and experimental results.
\end{abstract}

\section{Introduction}

Diluted magnetic semiconductors (DMSs) ${ }^{1}$ are attracting increasing attention in the physics community due to recent predictions $^{2,3}$ and reports ${ }^{4-6}$ of room-temperature ferromagnetism in some of these materials. Ferromagnetic DMSs ${ }^{4-7}$ have been proposed as pivotal components in a new category of spinbased electronics (spintronics) $)^{8,9}$ devices that aim to control

$\dagger$ University of Washington.

$\doteqdot$ Pacific Northwest National Laboratory.

(1) (a) Furdyna, J. K. J. Appl. Phvs. 1988, 64, R29-R64. (b) Furdyna, J. K., Kossut, J., Eds. Diluted Magnetic Semiconductors; Academic: New York, 1988; Vol. 25.

(2) Dietl, T.; Ohno, H.; Matsukura, F.; Cibert, J.; Ferrand, D. Science 2000 , 287, 1019-1022.

(3) Sato, K.; Katayama-Yoshida, H. Phys. Status Solidi B 2002, 229, 673680.

(4) Matsumoto, Y.; Murakami, M.; Shono, T.; Hasegawa, T.; Fukumura, T.; Kawasaki, M.; Ahmet, P.; Chikyow, T.; Koshihara, S.; Koinuma, H. Science 2001, 291, 854-856.

(5) For recent reviews, see: Chambers, S. A., Yoo, Y. K., Eds. MRS Bull. 2003, 23, 706-748.

(6) Pearton, S. J.; Abernathy, C. R.; Overberg, M. E.; Thaler, G. T.; Norton, D. P.; Theodoropoulou, N.; Hebard, A. F.; Park, Y. D.; Ren, F.; Kim, J.; Boatner, L. A. J. Appl. Phys. 2003, 93, 1-13.

(7) (a) Kacman, P. Semicond. Sci. Technol. 2001, 16, R25-R39. (b) Blinowski, J.; Kacman, P.; Dietl, T. Condens. Matter 2002, arXiv: cond-mat/0201012.

(8) (a) Wolf, S. A.; Awschalom, D. D.; Buhrman, R. A.; Daughton, J. M. von Molnár, S.; Roukes, M. L.; Chthelkanova, A. Y.; Treger, D. M. Science 2001, 294, 1488-1495. (b) Awschalom, D. D.; Flatté, M. E.; Samarth, N. Sci. Am. 2002, 286, 66-73. (c) Fiederling, R.; Keim, M.; Reuscher, G.; Ossau, W.; Schmidt, G.; Waag, A.; Molenkamp, L. W. Nature 1999, 402, 787-790. (d) Jonker, B. T.; Park, Y. D.; Bennett, B. R.; Cheong, H. D.; Kioseoglou, G.; Petrou, A. Phys. Rev. B 2000, 62, 8180-8183.

(9) Chye, Y.; White, M. E.; Johnston-Halperin, E.; Gerardot, B. D.; Awschalom, D. D.; Petroff, P. M. Phys. Rev. B 2002, 66, 201301/201301-201304.

10.1021/ja048427j CCC: $\$ 27.50$ @ 2004 American Chemical Society electron spin currents as well as charge currents to increase data processing speeds, reduce power consumption, reduce hardware dimensions, and possibly introduce new functionalities to semiconductor information processing technologies.

Theoreticians have identified $\mathrm{ZnO}$ as an excellent candidate host semiconductor for supporting high-Curie-temperature (high$T_{\mathrm{C}}$ ) ferromagnetism when doped with a variety of $3 \mathrm{~d}$ transition metal ions, particularly $\mathrm{Mn}^{2+}{ }^{2,3}$ Experimentalists have verified these predictions in some cases, with ferromagnetism above room temperature reported for thin films of $\mathrm{ZnO}$ doped with $\mathrm{Co}^{2+},{ }^{10} \mathrm{Co}^{2+} / \mathrm{Fe}^{2+}, 11$ and $\mathrm{V}^{2+},{ }^{12}$ prepared by vacuum deposition methods such as pulsed laser deposition (PLD). These findings remain controversial, however, and several laboratories have claimed to observe ferromagnetism arising only from phasesegregated impurities and not from the DMSs themselves. ${ }^{13,14}$ Not until very recently was ferromagnetism above room temperature reported for $\mathrm{Mn}^{2+}: \mathrm{ZnO},{ }^{15}$ even though this DMS was specifically highlighted in theoretical studies for its high$T_{\mathrm{C}}$ ferromagnetism potential. ${ }^{2,3}$ Many earlier studies of

(10) Ueda, K.; Tabata, H.; Kawai, T. Appl. Phvs. Lett. 2001, 79, 988-990.

(11) Cho, Y. M.; Choo, W. K.; Kim, H.; Kim, D.; Ihm, Y. Appl. Phys. Lett. 2002, 80, 3358-3360.

(12) Saeki, H.; Tabata, H.; Kawai, T. Solid State Commun. 2001, 120, 439443.

(13) Park, J. H.; Kim, M. G.; Jang, H. M.; Ryu, S.; Kim, Y. M. Appl. Phys. Lett. 2004, 84, 1338-1340.

(14) Ando, K. Condens. Matter 2002, arXiv: cond-mat/0208010.

(15) Sharma, P.; Gupta, A.; Rao, K. V.; Owens, F. J.; Sharma, R.; Ahuja, R. Osorio Guillen, J. M.; Johansson, B.; Gehring, G. A. Nature Mater. 2003, 2, 673-677.

J. AM. CHEM. SOC. 2004, 126, 9387-9398 -9387 
$\mathrm{Mn}^{2+}: \mathrm{ZnO}$ revealed only paramagnetism, or at best ferromagnetism below room temperature, with $T_{\mathrm{C}}$ ranging from 37 to $250 \mathrm{~K}$, and many have low saturation moments indicative of only partial magnetic ordering. ${ }^{10,16-18}$ The wide range of magnetic properties displayed by this and other ZnO DMSs prepared by apparently similar methods ${ }^{10,19}$ suggests that the criteria necessary for ferromagnetism are highly sensitive to the preparation conditions and ultimately to the materials composition. Developing reproducible methods for preparing high- $T_{\mathrm{C}}$ ferromagnetic DMSs is essential for their use in spintronics technologies but remains a central challenge in this field. When this challenge is addressed, the understanding of the fundamental origins of this interesting magnetic behavior will also be advanced.

Although ferromagnetism in DMSs has attracted attention primarily from physicists, we envision chemistry as offering several advantages in this research area. We speculate that direct chemical syntheses of $\mathrm{ZnO}$ DMSs can provide better control over materials composition than is obtained with some hightemperature vacuum deposition and solid-state synthetic techniques, which often use dopant source materials that are themselves undesirable contaminants (e.g., manganese oxides for $\mathrm{Mn}^{2+}: \mathrm{ZnO},{ }^{15,17} \mathrm{NiO}$ for $\mathrm{Ni}^{2+}: \mathrm{ZnO},{ }^{20}$ and cobalt oxides or Co metal for $\mathrm{Co}^{2+}: \mathrm{ZnO}^{21}$ ) or require high temperatures and reducing conditions that may promote segregation of metallic precipitates. ${ }^{13,18}$ The advantage of the direct chemical approach is illustrated in the case of $\mathrm{Ni}^{2+}: \mathrm{ZnO}$. The solid solubility of $\mathrm{Ni}^{2+}$ in $\mathrm{ZnO}$ is very low, ${ }^{22}$ and there is a large driving force for phase segregation. $\mathrm{Ni}^{2+}: \mathrm{ZnO}$ prepared by $\mathrm{PLD}$ using $\mathrm{NiO}$ as the source of $\mathrm{Ni}^{2+}$ showed a magnetic hysteresis only below $5 \mathrm{~K}^{20}$ that resembled the characteristic magnetic behavior of nanoscale $\mathrm{NiO},{ }^{23}$ raising concerns over the possibility of nanoscale $\mathrm{NiO}$ contaminants. In contrast, $\mathrm{Ni}^{2+}: \mathrm{ZnO}$ DMSs prepared from ionic solutions showed ferromagnetism above room temperature $\left(T_{\mathrm{C}}>350 \mathrm{~K}\right)$ that was demonstrably an intrinsic property of the $\mathrm{Ni}^{2+}: \mathrm{ZnO}$ DMS. ${ }^{24}$

In this paper we report the direct chemical synthesis of highquality colloidal $\mathrm{Mn}^{2+}: \mathrm{ZnO}$ quantum dots (QDs) and the use of these nanocrystals as solution-phase precursors for the preparation of nanocrystalline thin films by spin-coat processing. Many proposed spintronics devices involve DMSs of nanometer dimensions,${ }^{8}$ and solution syntheses provide the opportunity to study free-standing DMS crystals in this size regime. These

(16) (a) Norton, D. P.; Pearton, S. J.; Hebard, A. F.; Theodoropoulou, N.; Boatner, L. A.; Wilson, R. G. Appl. Phys. Lett. 2003, 82, 239-241. (b) Yoon, S. W.; Cho, S.-B.; We, S. C.; Yoon, S.; Suh, B. J.; Song, H. K.; Shin, Y. J. J. Appl. Phys. 2003, 93, 7879-7881.

(17) Fukumura, T.; Jin, Z.; Ohtomo, A.; Koinuma, H.; Kawasaki, M. Appl. Phvs. Lett. 1999, 75, 3366-3368.

(18) Theodoropoulou, N. A ; Hebard, A. F ; Norton, D. P.; Budai, J. D.; Boatner, L. A.; Lee, J. S.; Khim, Z. G.; Park, Y. D.; Overberg, M. E.; Pearton, S J.; Wilson, R. G. Solid State Electron. 2003, 47, 2231-2235.

(19) Jin, Z.-W.; Fukumura, T.; Hasegawa, K.; Yoo, Y.-Z.; Ando, K.; Sekiguchi, T.; Ahmet, P.; Chikyow, T.; Hasegawa, T.; Koinuma, H.; Kawasaki, M. J. Cryst. Growth 2002, 237-239, 548-552.

(20) Wakano, T.; Fujimura, N.; Morinaga, Y.; Abe, N.; Ashida, A.; Ito, T. Physica E 2001, 10, 260-264.

(21) (a) Lim, S.-W.; Hwang, D.-K.; Myoung, J.-M. Solid State Commun. 2003 , 125, 231-235. (b) Rode, K.; Anane, A.; Mattana, R.; Contour, J.-P.; Durand, O.; LeBourgeois, R. J. Appl. Phys. 2003, 93, 7676-7678.

(22) Bates, C. H.; White, W. B.; Roy, R. J. Inorg. Nucl. Chem. 1966, 28, 397405

(23) Bødker, F.; Hansen, M. F.; Bender Kotch, C.; Mørup, S. J. Magn. Magn. Mater. 2000, 221, 32-36. Cordente, N.; Toustou, B.; Colliere, V.; Amiens, C.; Chaudret, B.; Verelst, M.; Respaud, M.; Broto, J.-M. C. R. Acad. Sci. Paris, Chem. 2001, 4, 143-148.

(24) Radovanovic, P. V.; Gamelin, D. R. Phys. Rev. Lett. 2003, 91, 157202/ $1-4$. syntheses can be scaled up easily to produce gram quantities of the desired DMS, which greatly facilitates rapid exploration of the physical properties of these materials. Colloidal nanocrystals also have accessible surface chemistry that allows functionalization and processing in a variety of solvents, and this opens the door to potential building-block applications in nanotechnology, an area in which self-assembly is of increasing importance. Colloidal $\mathrm{Mn}^{2+}$-doped DMS nanocrystals of $\mathrm{ZnS},{ }^{25}$ $\mathrm{CdS},{ }^{26} \mathrm{ZnSe},{ }^{27} \mathrm{CdSe},{ }^{28} \mathrm{PbSe},{ }^{29}$ and $\mathrm{InAs}{ }^{30}$ have been reported previously. The synthesis and characterization of colloidal $\mathrm{Co}^{2+}$ and $\mathrm{Ni}^{2+}$-doped $\mathrm{ZnO}$ DMS-QDs ${ }^{24,31,32}$ and nanocrystalline $\mathrm{Mn}^{2+}: \mathrm{ZnO}$ powders prepared from solution ${ }^{33}$ have also been described recently. While this paper was under review, a report of $\mathrm{Mn}^{2+}$-doped $\mathrm{ZnO}$ colloids appeared. ${ }^{34} \mathrm{X}$-ray absorption and EPR spectroscopies were used to demonstrate that $\geq \sim 45 \%$ of the manganese was segregated at the nanocrystal surfaces in this preparation.

The synthesis of $\mathrm{Mn}^{2+}: \mathrm{ZnO}$ nanocrystals in this study was monitored using electron paramagnetic resonance (EPR) and electronic absorption spectroscopies. A method for removing $\mathrm{Mn}^{2+}$ ions from the nanocrystal surfaces is presented, and its efficacy is demonstrated by EPR spectroscopy. Simulations of the experimental X- and Q-band EPR spectra confirm substitutional doping of the $\mathrm{Mn}^{2+}$ ions in $\mathrm{ZnO}$ with very homogeneous speciation and with bulk-like $\mathrm{Mn}^{2+}$ ground-state electronic structures. These colloidal DMS nanocrystals represent highly versatile solution-phase building blocks that are compatible with numerous standard solution processing methods. We report robust, high- $T_{\mathrm{C}}$ ferromagnetism in thin films of these nanocrystals prepared by spin-coating, with $300 \mathrm{~K}$ saturation moments as high as $1.35 \mu_{\mathrm{B}} / \mathrm{Mn}^{2+}$, nearly an order of magnitude higher than that previously reported. ${ }^{15}$ The $\mathrm{Mn}^{2+}: \mathrm{ZnO}$ ferromagnetism is accompanied by a broad ferromagnetic resonance EPR signal that reflects the high multiplicity of the ferromagnetic ground state. The preparation of more complex ferromagnetic semiconductor nanoarchitectures by solution processing methods may therefore be envisioned.

\section{Experimental Section}

A. Materials. Zinc acetate dihydrate $\left(\mathrm{Zn}(\mathrm{OAc})_{2} \cdot 2 \mathrm{H}_{2} \mathrm{O}, 98 \%\right.$, $<0.0005 \%$ magnetic impurities, Strem), manganese acetate tetrahydrate $\left(\mathrm{Mn}(\mathrm{OAc})_{2} \cdot 4 \mathrm{H}_{2} \mathrm{O}, 99.999 \%\right.$, Strem $)$, manganese nitrate hydrate $\left(\mathrm{Mn}\left(\mathrm{NO}_{3}\right)_{2} \cdot x \mathrm{H}_{2} \mathrm{O}, 98 \%\right.$, Sigma-Aldrich), tetramethylammonium hydroxide $\left(\mathrm{N}\left(\mathrm{CH}_{4}\right)_{4} \mathrm{OH} \cdot 5 \mathrm{H}_{2} \mathrm{O}, 97 \%\right.$, Sigma-Aldrich), dodecylamine $\left(\mathrm{CH}_{3}\left(\mathrm{CH}_{2}\right)_{11} \mathrm{NH}_{2}, 98 \%\right.$, Sigma-Aldrich), trioctylphosphine oxide (TOPO, $\left(\mathrm{CH}_{3}\left(\mathrm{CH}_{2}\right)_{7}\right)_{3} \mathrm{P}(\mathrm{O}), 90 \%$, Sigma-Aldrich), dimethyl sulfoxide (DMSO, 99.7\%, Acros), and absolute ethanol (AAPER) were purchased and used as received.

(25) Bol, A. A.; Meijerink, A. Phvs. Rev. B 1998, 58, 15997-16000

(26) Hoffman, D. M.; Meyer, B. K.; Ekimov, A. I.; Merkulov, I. A.; Efros, A L.; Rosen, M.; Counio, G.; Gacoin, T.; Boilot, J.-P. Solid State Commun. 2000, 114, 547-550.

(27) Norris, D. J.; Yao, N.; Charnock, F. T.; Kennedy, T. A. Nano Lett. 2001, $1,3-7$.

(28) Mikulec, F. V.; Kuno, M.; Bennati, M.; Hall, D. A.; Griffin, R. G.; Bawendi, M. G. J. Am. Chem. Soc. 2000, 122, 2532-2540.

(29) Ji, T.; Jian, W.-B.; Fang, J. J. Am. Chem. Soc. 2003, 125, 8448-8449.

(30) Stowell, C. A.; Wiacek, R. J.; Saunders, A. E.; Korgel, B. A. Nano Lett. 2003, 3, 1441-1447.

(31) Radovanovic, P. V.; Norberg, N. S.; McNally, K. E.; Gamelin, D. R. J. Am. Chem. Soc. 2002, 124, 15192-15193.

(32) Schwartz, D. A.; Norberg, N. S.; Nguyen, Q. P.; Parker, J. M.; Gamelin, D. R. J. Am. Chem. Soc. 2003, 125, 13205-13218.

(33) Zhou, H.; Hofmann, D. M.; Hofstaetter, A.; Meyer, B. K. J. Appl. Phys. 2003, 94, 1965-1968.

(34) Viswanatha, R.; Sapra, S.; Gupta, S. S.; Satpati, B.; Satyam, P. V.; Dev, B.; Sarma, D. D. J. Phys. Chem. B 2004, 108, 6303-6310. 
B. Sample Preparation. $\mathrm{Mn}^{2+}: \mathrm{ZnO}$ nanocrystals were synthesized at room temperature by dropwise addition of 1.7 equiv of an ethanolic solution of $0.55 \mathrm{M}$ tetramethylammonium hydroxide $\left(\mathrm{N}(\mathrm{Me})_{4} \mathrm{OH} \cdot 5 \mathrm{H}_{2} \mathrm{O}\right)$ to a $0.10 \mathrm{M}$ solution of $x \mathrm{Mn}(\mathrm{OAc})_{2} \cdot 4 \mathrm{H}_{2} \mathrm{O} /(1-x) \mathrm{Zn}(\mathrm{OAc})_{2} \cdot 2 \mathrm{H}_{2} \mathrm{O}$ dissolved in DMSO under constant stirring. Following initial rapid growth from solution that proceeds on the time scale of minutes, ${ }^{32}$ growth of the nanocrystals continues by Ostwald ripening on the time scale of several days at room temperature or can be accelerated by heating the solution to ca. $60{ }^{\circ} \mathrm{C}$. After growth, the $\mathrm{Mn}^{2+}: \mathrm{ZnO}$ nanocrystals were precipitated by addition of ethyl acetate and resuspended in ethanol. Iterative washing by precipitation with heptane and resuspension in ethanol ensures that excess reactants have been removed from the product. After being washed, the nanocrystals suspended in ethanol were capped with dodecylamine and resuspended in toluene. A sample in which $\mathrm{Mn}^{2+}$ ions were deliberately bound to the surfaces of $\mathrm{ZnO}$ nanocrystals was prepared by first synthesizing pure $\mathrm{ZnO}$ nanocrystals following the above procedure with no added $\mathrm{Mn}(\mathrm{OAc})_{2}$. After the nanocrystals were washed and resuspended in ethanol, a small amount of $\mathrm{Mn}(\mathrm{OAc})_{2}$ (ca. $2 \%$ of $\mathrm{Zn}^{2+}$ ) was mixed into the $\mathrm{ZnO}$ suspension, and 0.002 equiv of an ethanolic solution of $\mathrm{LiOH}$ was added dropwise. The resulting surface-bound $\mathrm{Mn}^{2+}: \mathrm{ZnO}$ nanocrystals were subsequently washed, capped with dodecylamine, and resuspended in toluene for EPR experiments.

To remove $\mathrm{Mn}^{2+}$ ions from the nanocrystal surfaces, dodecylaminecapped nanocrystals were heated in dodecylamine $\left(\mathrm{mp} \mathrm{ca} .30^{\circ} \mathrm{C}\right)$ at $180{ }^{\circ} \mathrm{C}$ for ca. $30 \mathrm{~min}$ under nitrogen. The nanocrystals were allowed to cool to below $80{ }^{\circ} \mathrm{C}$ and were then precipitated and washed with ethanol. The resulting amine-treated powders were resuspended in toluene or other nonpolar solvents to form colloidal suspensions of high optical quality that are stable for several months. In some experiments, $\mathrm{Mn}^{2+}$ ions were removed from the nanocrystal surfaces by heating in technical grade trioctylphosphine oxide (TOPO) following a procedure described previously. ${ }^{32}$

Nanocrystalline thin films of $\mathrm{Mn}^{2+}: \mathrm{ZnO}$ were prepared by spincoating amine-treated $\mathrm{Mn}^{2+}: \mathrm{ZnO}$ colloids onto $1 \mathrm{~cm} \times 0.5 \mathrm{~cm}$ fusedsilica substrates. The films were annealed at $525^{\circ} \mathrm{C}$ for $2 \mathrm{~min}$ in air after each spin-coated layer was added. Film A comprised 40 coats and had a mass of $0.59 \mathrm{mg}$. Films B and C were made from 20 coats each and had masses of 0.24 and $0.28 \mathrm{mg}$, respectively.

C. Physical Characterization. X-band $(9.34 \mathrm{GHz})$ electron paramagnetic resonance (EPR) spectra were collected on a Bruker EMX EPR spectrometer at the University of Washington, and Q-band (34.0 $\mathrm{GHz}$ ) EPR spectra were collected on a Bruker ESP300E EPR spectrometer at the Pacific Northwest National Laboratories. The X- and Q-band EPR data for the free-standing nanocrystals were recorded at room temperature on colloids suspended in toluene. Room-temperature X-band EPR spectra of thin films were measured by attaching the films to EPR tubes with nonmagnetic tape and aligning them perpendicular to the magnetic field in the EPR cavity. Simulations of the X- and Q-band EPR spectra were performed using a full-matrix diagonalization routine implemented by SIM (ver.2002), written and graciously provided by Prof. Høgni Weihe of the University of Copenhagen.

Absorption spectra of free-standing nanocrystals were measured at room temperature on colloids using $1 \mathrm{~cm}$ path length cuvettes using a Cary 500E (Varian) spectrophotometer. To observe both weak and intense absorption features during a base titration experiment, a portion of the sample was extracted from the reaction vessel, measured, diluted by a constant factor $(70 \times)$, and then remeasured. Low-temperature MCD spectra were collected on drop-coated films (frozen solutions) as described previously. ${ }^{32} \mathrm{MCD}$ intensities were measured as the differential absorbance $\Delta A=A_{\mathrm{L}}-A_{\mathrm{R}}$ (where $\mathrm{L}$ and $\mathrm{R}$ refer to left and right circularly polarized photons) and are reported as $\theta(\mathrm{deg})=$ $32.9 \Delta A / A$. Colloid luminescence spectra were measured in $1 \mathrm{~cm} \times 1$ $\mathrm{cm}$ fluorescence cuvettes on a Jobin Yvon FluoroMax-2 fluorimeter.

$\mathrm{Cu} \mathrm{K} \alpha \mathrm{X}$-ray powder diffraction data were collected on a Philips PW 1830 X-ray diffractometer for powders and on a Rigaku Rotaflex

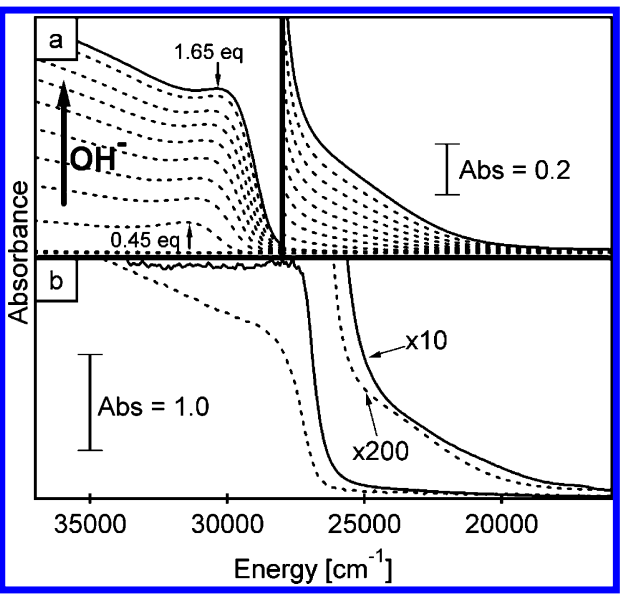

Figure 1. (a) $300 \mathrm{~K}$ absorption spectra of a $0.10 \mathrm{M}$ DMSO solution of $2 \%$ $\mathrm{Mn}(\mathrm{OAc})_{2} / 98 \% \mathrm{Zn}(\mathrm{OAc})_{2}$ collected following successive additions of 0.15 equiv of $0.55 \mathrm{M} \mathrm{N}(\mathrm{Me})_{4} \mathrm{OH}$ in ethanol. The solid line shows the data collected after 1.65 equiv of base was added. (b) $300 \mathrm{~K}$ absorption spectra of (- -) colloidal $0.20 \% \mathrm{Mn}^{2+}: \mathrm{ZnO}$ nanocrystals after treatment with dodecylamine and $(-)$ a thin film prepared by spin-coating the same nanocrystals onto fused silica (film A). The thin-film absorbance reaches the stray light limit at ca. $27000 \mathrm{~cm}^{-1}$.

RTP300 diffractometer for thin films. High-resolution transmission electron microscopy (HRTEM) images were collected at the Pacific Northwest National Laboratories on a JEOL 2010 transmission electron microscope $(200 \mathrm{kV})$ with a high-brightness $\mathrm{LaB}_{6}$ filament as an electron source. Dopant concentrations were determined by inductively coupled plasma atomic emission spectrometry (ICP-AES, Jarrel Ash model 955).

Magnetic susceptibility data for $\mathrm{Mn}^{2+}: \mathrm{ZnO}$ nanocrystalline powders rapidly precipitated from toluene and for spin-coated thin films were collected using a Quantum Design MPMS-5S SQUID magnetometer. All data were corrected for the diamagnetism of the substrate and sample holder.

\section{Results}

Figure 1a shows electronic absorption spectra of a $0.10 \mathrm{M}$ DMSO solution of $2 \% \mathrm{Mn}(\mathrm{OAc})_{2} \cdot 4 \mathrm{H}_{2} \mathrm{O} / 98 \% \mathrm{Zn}(\mathrm{OAc})_{2} \cdot 2 \mathrm{H}_{2} \mathrm{O}$ collected following successive additions of 0.15 equiv of 0.55 $\mathrm{M} \mathrm{N}(\mathrm{Me})_{4} \mathrm{OH}$ in ethanol. An intense absorption feature appears at ca. $30000 \mathrm{~cm}^{-1}$ after addition of 0.45 equiv of base that is readily identified as the first excitonic band gap transition of $\mathrm{ZnO} .^{35,36}$ Further base addition yields an approximately linear increase in band gap absorbance. A sub-bandgap feature at $24000 \mathrm{~cm}^{-1}$ also appears after 0.45 equiv of base has been added, and its intensity grows with continued addition of base, giving the colloidal suspension a brown color. This sub-bandgap feature has been observed previously in other $\mathrm{Mn}^{2+}: \mathrm{ZnO}$ preparations. ${ }^{17,22,37,38}$ The solid line in Figure 1a shows the spectrum collected after 1.65 equiv of base was added. Base addition beyond ca. 1.65 equiv caused the suspensions to begin to cloud.

Figure 1b shows absorption spectra of $0.20 \% \mathrm{Mn}^{2+}: \mathrm{ZnO}$ colloids after heating of the colloids in dodecylamine to remove $\mathrm{Mn}^{2+}$ ions from the nanocrystal surfaces (vide infra). These nanocrystals, prepared with an initial dopant level of $0.50 \%$ $\mathrm{Mn}^{2+}$, were found by ICP-AES to contain $0.20 \pm 0.01 \% \mathrm{Mn}^{2+}$

(35) Spanhel, L.; Anderson, M. A. J. Am. Chem. Soc. 1991, 113, 2826-2833. (36) Meulenkamp, E. A. J. Phys. Chem. B 1998, 102, 5566-5572.

(37) Jin, Z.-W.; Yoo, Y.-Z.; Sekiguchi, T.; Chikyow, T.; Ofuchi, H.; Fujioka, H.; Oshima, M.; Koinuma, H. Appl. Phys. Lett. 2003, 83, 39-41.

(38) Fichou, D.; Pouliquen, J.; Kossanyi, J.; Jakani, M.; Campet, G.; Claverie, J. J. Electroanal. Chem. 1985, 188, 167-187. 


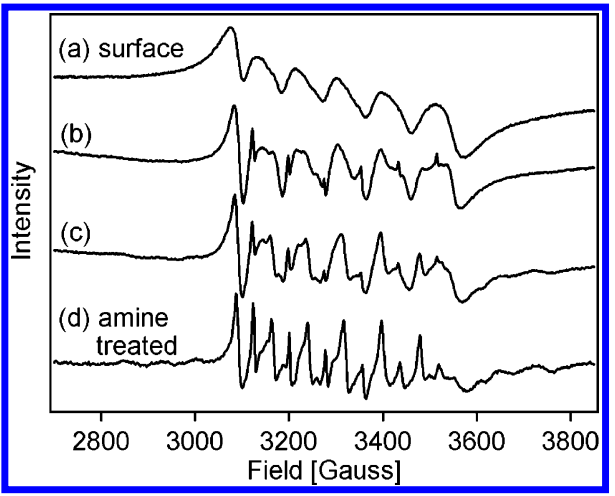

Figure 2. $300 \mathrm{~K} \mathrm{X}$-band EPR spectra of colloidal $\mathrm{Mn}^{2+}: \mathrm{ZnO}$ nanocrystals. (a) Surface-bound $\mathrm{Mn}^{2+}: \mathrm{ZnO}$ nanocrystals. Samples prepared from $0.02 \%$ $\mathrm{Mn}^{2+} / 99.98 \% \mathrm{Zn}^{2+}$ reaction solution collected (b) $10 \mathrm{~min}$ after base addition, (c) after $2 \mathrm{~h}$ of heating at $60^{\circ} \mathrm{C}$, and (d) after treatment with dodecylamine.

(i.e., $\mathrm{Zn}_{0.998} \mathrm{Mn}_{0.002} \mathrm{O}$ ). Figure $1 \mathrm{~b}$ also shows the absorption spectrum of a thin film prepared by spin-coating the same colloids onto a fused silica substrate. We refer to this film throughout this paper as film A. The band gap energy of the nanocrystalline thin film is similar to that of bulk $\mathrm{ZnO}$ (ca. $27000 \mathrm{~cm}^{-1}$ at $\left.300 \mathrm{~K}\right){ }^{6}$ The band gap energy of the colloids determined by the same method is slightly larger, attributable to quantum confinement. The sub-bandgap absorption feature is observed in both the colloids and the thin film.

Figure 2 shows room-temperature X-band EPR spectra of colloidal $\mathrm{Mn}^{2+}: \mathrm{ZnO}$ nanocrystals in toluene. Figure $2 \mathrm{a}$ is the spectrum of $\mathrm{ZnO}$ nanocrystals with $\mathrm{Mn}^{2+}$ ions deliberately bound to the surfaces. Figure $2 b-d$ shows EPR spectra of aliquots removed from a reaction in which 1.7 equiv of $0.55 \mathrm{M}$ $\mathrm{N}(\mathrm{Me})_{4} \mathrm{OH}$ was added to a $0.10 \mathrm{M}$ DMSO solution of $0.02 \%$ $\mathrm{Mn}(\mathrm{OAc})_{2} / 99.98 \% \mathrm{Zn}(\mathrm{OAc})_{2}$. Samples for EPR measurements were removed from the reaction (b) 10 min after base addition at room temperature, (c) after nanocrystal growth was allowed for $2 \mathrm{~h}$ at $60{ }^{\circ} \mathrm{C}$, and (d) after the nanocrystalline product was washed and heated in dodecylamine for $30 \mathrm{~min}$ at $180{ }^{\circ} \mathrm{C}$. All samples were washed and capped with dodecylamine. The average nanocrystal diameters for (b) and (c) were estimated from the nanocrystal band gap absorption energies ${ }^{36}$ to be 4.0 and $5.6 \mathrm{~nm}$, respectively. All spectra show six main features, characteristic of the hyperfine coupling of $\mathrm{Mn}^{2+}(I=5 / 2)$. The spectra evolve from having six broad signals with an apparent hyperfine splitting of ca. $88 \mathrm{G}$ (Figure 2a) to showing extensive resolved fine structure with narrow features and an apparent hyperfine splitting of ca. $78 \mathrm{G}$ for the major features (Figure 2d).

Figure 3a presents powder X-ray diffraction (XRD) data for the $0.20 \% \mathrm{Mn}^{2+}: \mathrm{ZnO}$ nanocrystals shown in Figure $1 \mathrm{~b}$, precipitated rapidly from toluene (dashed line), and for film A (solid line), prepared by spin-coating the same nanocrystals onto fused silica. All of the peaks in the two diffraction patterns match those of wurtzite $\mathrm{ZnO}$, shown as the indexed lines in the bottom of Figure 3a. TEM data collected for the same nanocrystals are shown in Figure $3 b-d$. The images in Figure $3 b, d$ show approximately spherical and highly crystalline nanocrystals. The characteristic lattice spacings of wurtzite $\mathrm{ZnO}$ are readily identified in the high-resolution TEM images of these nanocrystals (Figure 3d). Analysis of ca. 100 nanocrystals (Figure 3c) yields an average crystal diameter of $6.1 \pm 0.7 \mathrm{~nm}$.

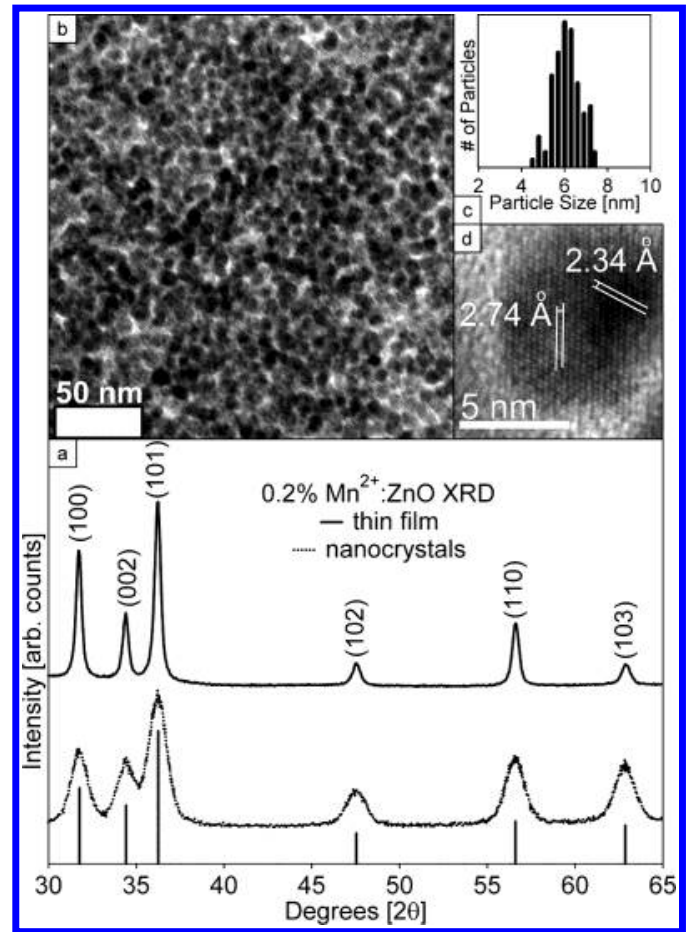

Figure 3. (a) Powder X-ray diffraction data: (*) $0.20 \% \quad \mathrm{Mn}^{2+}: \mathrm{ZnO}$ nanocrystals; (-) thin film prepared from the $0.20 \% \mathrm{Mn}^{2+}: \mathrm{ZnO}$ nanocrystals. Peak positions for wurtzite $\mathrm{ZnO}$ are included for reference. (b) Overview TEM image of $0.20 \% \mathrm{Mn}^{2+}: \mathrm{ZnO}$ nanocrystals. (c) Histogram of 100 crystal diameters $(6.1 \pm 0.7 \mathrm{~nm}$ average diameter). (d) High-resolution TEM image of a single nanocrystal showing lattice spacings matching those of $\mathrm{ZnO}$.

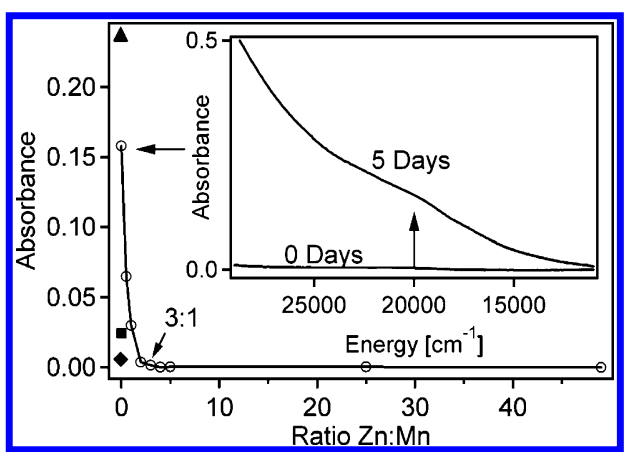

Figure 4. Absorption intensities of various solutions of $0.002 \mathrm{M} \mathrm{Mn}(\mathrm{OAc})_{2}$ in DMSO measured at $20000 \mathrm{~cm}^{-1}$ after 5 days in air. (O) Solutions with varying ratios of $\mathrm{Zn}(\mathrm{OAc})_{2}$ to $\mathrm{Mn}(\mathrm{OAc})_{2}$. (ム) A solution with a 5:1 ratio of $\mathrm{Na}(\mathrm{OAc})$ to $\mathrm{Mn}(\mathrm{OAc})_{2}$. (莐 An anaerobic solution of $\mathrm{Mn}(\mathrm{OAc})_{2}$. ( ) A solution with $0.002 \mathrm{M} \mathrm{Mn}\left(\mathrm{NO}_{3}\right)_{2}$ instead of $\mathrm{Mn}(\mathrm{OAc})_{2}$. Inset: Absorption spectra of a DMSO solution of $0.002 \mathrm{M} \mathrm{Mn}(\mathrm{OAc})_{2}$ after mixing (0 days) and after 5 days in air.

Figure 4 shows absorption data collected for various solutions of $0.002 \mathrm{M} \mathrm{Mn}(\mathrm{OAc})_{2} \cdot 4 \mathrm{H}_{2} \mathrm{O}$ in DMSO that were allowed to stand open to the atmosphere for several days. This $\mathrm{Mn}^{2+}$ concentration is the same as that used for the synthesis of $2 \%$ $\mathrm{Mn}^{2+}: \mathrm{ZnO}$ nanocrystals. The inset shows the change in absorption of the $0.002 \mathrm{M} \mathrm{Mn}(\mathrm{OAc})_{2} \cdot 4 \mathrm{H}_{2} \mathrm{O}$ solution when the solution was exposed to air for 5 days. The solution turns brown, and a broad tailing absorption feature emerges. The open circles in Figure 4 show the absorption intensity at $20000 \mathrm{~cm}^{-1}$ after 5 days for solutions of $0.002 \mathrm{M} \mathrm{Mn}(\mathrm{OAc})_{2} \cdot 4 \mathrm{H}_{2} \mathrm{O}$ with varying amounts of $\mathrm{Zn}(\mathrm{OAc})_{2} \cdot 2 \mathrm{H}_{2} \mathrm{O}$ added. A sharp decrease in the formation of the brown absorption intensity is observed as the ratio of $\mathrm{Zn}^{2+}$ to $\mathrm{Mn}^{2+}$ increases, reaching almost zero at a ratio of $3 \mathrm{Zn}(\mathrm{OAc})_{2} \cdot 2 \mathrm{H}_{2} \mathrm{O}: 1 \mathrm{Mn}(\mathrm{OAc})_{2} \cdot 4 \mathrm{H}_{2} \mathrm{O}$. The absorption 


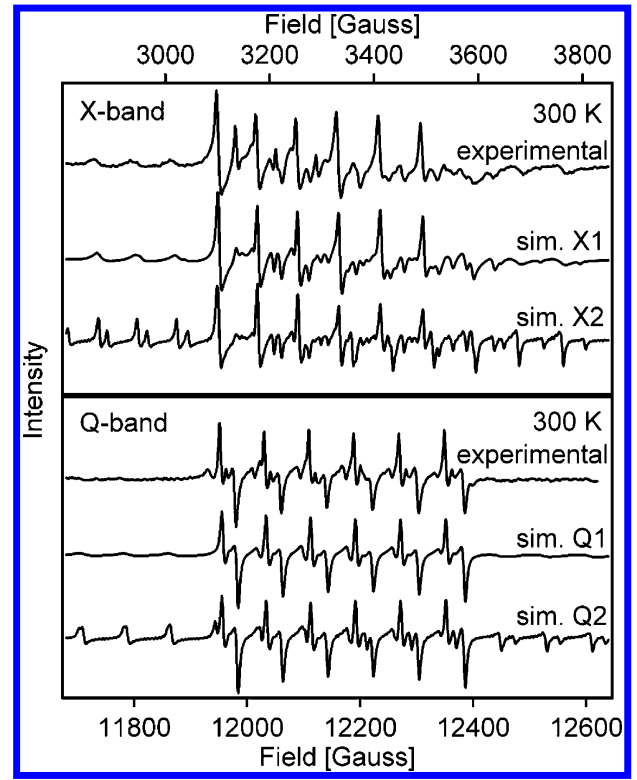

Figure 5. Experimental and simulated $300 \mathrm{~K} \mathrm{X}$ - and Q-band EPR spectra of colloidal dodecylamine-capped $0.02 \% \mathrm{Mn}^{2+}: \mathrm{ZnO}$ nanocrystals in toluene. Simulations with (X1 and Q1) and without (X2 and Q2) $\sigma=2 \% D$-strain are included.

intensities measured for analogous $0.002 \mathrm{M} \mathrm{Mn}(\mathrm{OAc})_{2} \cdot 4 \mathrm{H}_{2} \mathrm{O}$ solutions allowed to stand anaerobically ( $\mathbf{\square}$ ) or in air with 0.010 $\mathrm{M} \mathrm{NaOAc}$ added $(\boldsymbol{\Delta})$ are also included in Figure 4. Addition of $\mathrm{NaOAc}$ causes $50 \%$ more absorption intensity after 5 days, whereas the anaerobic solution shows only a small increase in absorption after 5 days. When $\mathrm{Mn}\left(\mathrm{NO}_{3}\right)_{2} \cdot x \mathrm{H}_{2} \mathrm{O}(\bullet)$ is used in place of $\mathrm{Mn}(\mathrm{OAc})_{2} \cdot 4 \mathrm{H}_{2} \mathrm{O}$, little or no absorbance change is observed after 5 days in air.

Room-temperature X- and Q-band EPR spectra of surfacecleaned $\mathrm{Mn}^{2+}: \mathrm{ZnO}$ colloidal nanocrystals prepared from a $0.02 \%$ $\mathrm{Mn}(\mathrm{OAc})_{2} \cdot 4 \mathrm{H}_{2} \mathrm{O} / 99.98 \% \mathrm{Zn}(\mathrm{OAc})_{2} \cdot 2 \mathrm{H}_{2} \mathrm{O}$ solution as in Figure $2 \mathrm{~d}$ are displayed in Figure 5. Both experimental spectra are highly resolved, exhibiting small peak widths for all features and clearly showing six major hyperfine lines. Many additional features are also present, however, including three outer peaks on either side of the main sextet in the X-band EPR spectrum.

Figure 6 shows $300 \mathrm{~K}$ absorption and $5 \mathrm{~K}$ MCD spectra of free-standing TOPO-capped $1.1 \% \mathrm{Mn}^{2+}: \mathrm{ZnO}$ nanocrystals. The $5 \mathrm{~K}$ MCD spectrum shows a broad negative pseudo- $\mathcal{A}$ term MCD feature associated with this sub-bandgap absorption that tails throughout the visible energy window (shown down to $16000 \mathrm{~cm}^{-1}$ ). The MCD intensity becomes negative again at $28000 \mathrm{~cm}^{-1}$ with the onset of the $\mathrm{ZnO}$ band edge. The pseudo- $\mathcal{A}$ term MCD intensity increases with increasing applied field and approaches saturation above ca. $4 \mathrm{~T}$ (Figure 6, inset).

Figure 7 shows room-temperature absorption and luminescence spectra of $\mathrm{ZnO}, 0.13 \% \mathrm{Mn}^{2+}: \mathrm{ZnO}$ (estimated concentration), and $1.3 \% \mathrm{Mn}^{2+}: \mathrm{ZnO}$ colloidal nanocrystals capped with dodecylamine and suspended in toluene. All three samples were heated with dodecylamine following the procedure described above for removal of surface-bound $\mathrm{Mn}^{2+}$. All luminescence spectra were collected with excitation into the band gap region (28 $200 \mathrm{~cm}^{-1}$ ). The absorption intensities at the excitation energies, which ranged between 0.1 and 0.4 absorbance unit, were normalized in Figure 7, and the emission intensities were changed proportionally. The pure $\mathrm{ZnO}$ nanocrystals show a broad visible luminescence band centered at ca. $18600 \mathrm{~cm}^{-1}$

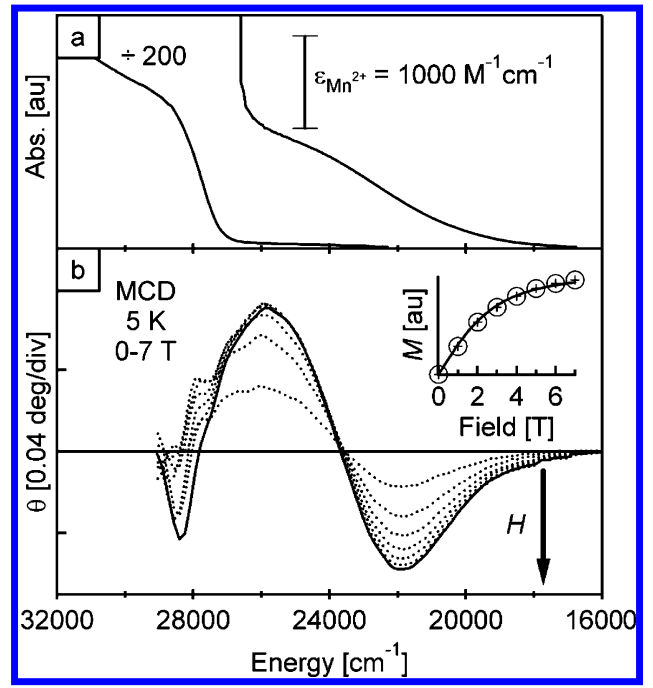

Figure 6. (a) $300 \mathrm{~K}$ absorption spectrum of colloidal TOPO-capped $1.1 \%$ $\mathrm{Mn}^{2+}: \mathrm{ZnO}$ nanocrystals. (b) Variable-field $5 \mathrm{~K}$ MCD spectra of the same nanocrystals, drop-coated onto quartz disks to form frozen solutions. The inset shows the $5 \mathrm{~K}$ MCD saturation magnetization probed at $21900 \mathrm{~cm}^{-1}$. The solid line shows the $S=5 / 2$ saturation magnetization at $5 \mathrm{~K}$ predicted by the Brillouin function (eq 4 ).

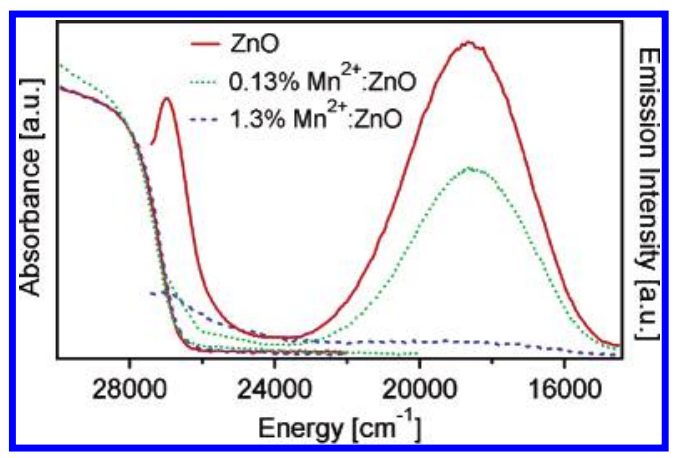

Figure 7. $300 \mathrm{~K}$ absorption and luminescence spectra of pure $\mathrm{ZnO}(-)$, $0.13 \% \mathrm{Mn}^{2+}: \mathrm{ZnO}$ (estimated concentration) $(\cdots)$, and $1.3 \% \mathrm{Mn}^{2+}: \mathrm{ZnO}$ (- - ) colloids.

and a relatively intense UV emission band at $26900 \mathrm{~cm}^{-1}$. The $0.13 \% \mathrm{Mn}^{2+}: \mathrm{ZnO}$ colloids show a similar luminescence spectrum, but the visible and UV emission intensities have been reduced by 42 and $69 \%$, respectively, relative to the pure $\mathrm{ZnO}$ nanocrystal spectrum. The visible emission in the $1.3 \%$ $\mathrm{Mn}^{2+}: \mathrm{ZnO}$ colloids is quenched by $96 \%$, and they also do not show the same excitonic emission feature in the UV but show only a weak intensity that may arise from scattering.

Figure 8 shows the magnetic susceptibilities of free-standing $0.20 \pm 0.01 \% \mathrm{Mn}^{2+}: \mathrm{ZnO}$ nanocrystals and three thin films (films A, B, and C) prepared by spin-coating the $0.20 \%$ $\mathrm{Mn}^{2+}: \mathrm{ZnO}$ nanocrystals onto fused silica substrates. Film A is the same film shown in Figure 1b. The magnetization of the free-standing nanocrystals is linear with small applied fields $(<1$ $\mathrm{T}$, or $10000 \mathrm{Oe}$ ) at $5 \mathrm{~K}$ and is strongly temperature dependent. In sharp contrast, the thin films all show rapid magnetic saturation and clear magnetic hystereses at both low and high temperatures. For each film, a temperature- and field-dependent magnetization signal similar to that of the free-standing nanocrystals is observed superimposed on the nearly temperatureindependent magnetic hysteresis. The $300 \mathrm{~K}$ saturation moments $\left(M_{\mathrm{S}}\right)$ for the three films are (A) 0.67 , (B) 1.18, and (C) 1.35 $\mu_{\mathrm{B}} / \mathrm{Mn}^{2+}$. The $300 \mathrm{~K}$ remanent magnetization $\left(M_{\mathrm{R}}\right)$ and coercivity $\left(H_{\mathrm{C}}\right)$ values are very similar for all three films, with 


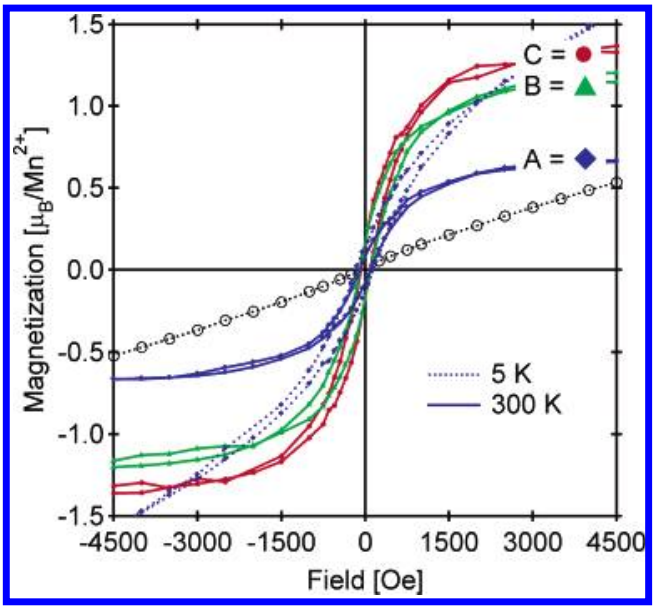

Figure 8. $5 \mathrm{~K}(\cdots)$ and $300 \mathrm{~K}(-)$ magnetic susceptibilities of $0.20 \% \mathrm{Mn}^{2+}: \mathrm{ZnO}$ nanocrystals $(\mathrm{O})$ and thin films $(\bullet$, film A; $\boldsymbol{\Delta}$, film B; $\bullet$, film C).

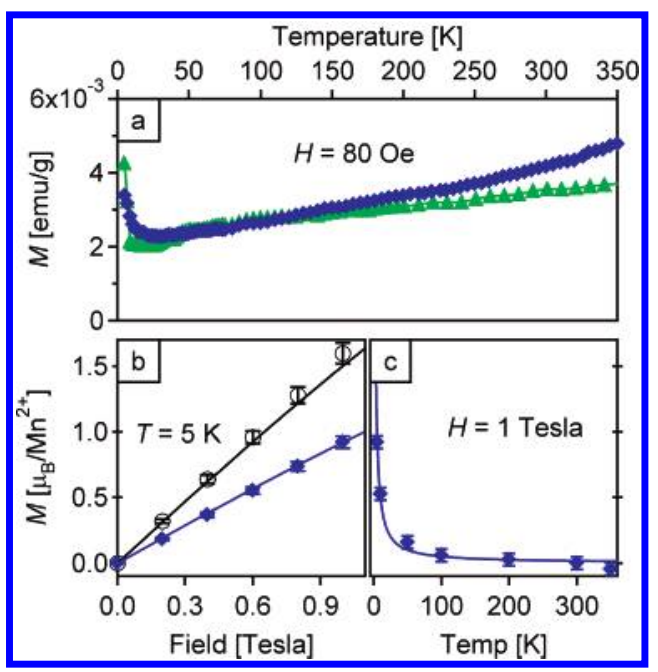

Figure 9. Magnetic susceptibility data for $0.20 \pm 0.01 \% \mathrm{Mn}^{2+}: \mathrm{ZnO}$. (a) Zero-field-cooled magnetization of films A $(\bullet)$ and B ( $\mathbf{\Delta})$ with an applied magnetic field of $80 \mathrm{Oe}$. (b) $5 \mathrm{~K}$ field-dependent magnetization of freestanding nanocrystals $(\mathrm{O})$ and of the residual paramagnetic magnetization of film A. (c) Temperature dependence of the residual paramagnetic magnetization of film A with $H=1 \mathrm{~T}$. The solid lines in (b) and (c) show the Brillouin function calculated using $S=5 / 2$ and $g=1.999$ (eq 4, see text). The error bars represent the uncertainty in $\mathrm{Mn}^{2+}$ concentration.

averages of $0.12 \pm 0.01 M_{\mathrm{S}}$, and $92 \pm 7 \mathrm{Oe}$, respectively. The magnetic hysteresis parameters for films $\mathrm{A}, \mathrm{B}$, and $\mathrm{C}$ are plotted as a function of temperature in Figure S2 of the Supporting Information. All three hysteresis parameters decrease gradually as the temperature is increased from $5 \mathrm{~K}$ to the instrument limit of $350 \mathrm{~K}$.

Zero-field-cooled (ZFC) magnetization measurements for films A and B from Figure 8 are shown in Figure 9a. Both films show a steep decrease in magnetization with increasing temperature between 5 and $10 \mathrm{~K}$, followed by a gradual increase in magnetization between 10 and $350 \mathrm{~K}$. Figure $9 \mathrm{~b}$ shows the $5 \mathrm{~K}$ linear magnetization signal for film $\mathrm{A}$, obtained by subtraction of the ferromagnetic signal from the $5 \mathrm{~K}$ data in Figure 8 . These data are compared quantitatively to the data collected for the free-standing nanocrystals, shown in Figure 8. The linear magnetization signal of the thin film is $64 \%$ as large as that of the free-standing nanocrystals. Figure 9c plots the temperature dependence of the $1 \mathrm{~T}$ magnetization for film A, obtained by subtracting the ferromagnetic signals from the

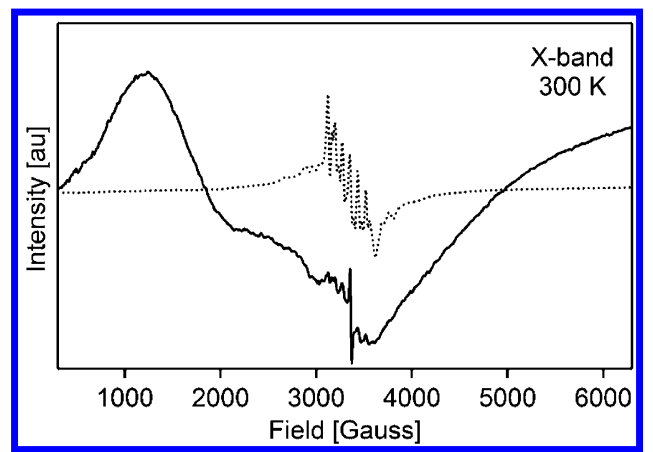

Figure 10. $300 \mathrm{~K}$ X-band EPR spectra. (-) Ferromagnetic $0.20 \%$ $\mathrm{Mn}^{2+}: \mathrm{ZnO}$ thin film (film A) from Figure $8 .(\cdots)$ The colloids from which film A was prepared, suspended in toluene.

data in Figure 8 and from analogous data collected at intermediate temperatures. These data show a strong decrease in magnetization with increasing temperature, reaching nearly zero at room temperature.

Figure 10 compares the $300 \mathrm{~K} \mathrm{X}$-band EPR spectra of film $\mathrm{A}$ and the free-standing $0.20 \% \mathrm{Mn}^{2+}: \mathrm{ZnO}$ colloids used to make this film. Whereas the colloid spectrum shows extensive hyperfine structure between 3000 and $3600 \mathrm{G}$, the thin-film EPR spectrum shows an intense, broad resonance spanning the entire spectral range. Hyperfine structure similar to that of the colloid spectrum can be observed superimposed on the broad feature in the spectrum of the thin film. Additionally, a new, sharp resonance at $H=3362 \mathrm{G}(g=2.00)$ is observed in the thin film.

\section{Analysis and Discussion}

A. Manganese Incorporation during Growth of $\mathrm{ZnO}$ Nanocrystals. The synthesis of $\mathrm{Mn}^{2+}$-doped $\mathrm{ZnO}$ nanocrystals was monitored by electronic absorption spectroscopy. The absorption spectra in Figure 1 show the characteristic $\mathrm{ZnO}$ band gap absorbance at ca. $30000 \mathrm{~cm}^{-1}$ upon addition of base to the DMSO solution of $\mathrm{Zn}^{2+}$, but only after 0.45 equiv of base has been added. This induction period reflects the buildup of $\mathrm{Zn}^{2+}$ precursor concentrations toward supersaturation prior to $\mathrm{ZnO}$ nucleation, as described by the LaMer model. ${ }^{39}$ The precursors for $\mathrm{ZnO}$ nucleation under these conditions are believed to be basic zinc acetate clusters. ${ }^{35,40}$ After 0.45 equiv, subsequent base addition causes further nucleation of $\mathrm{ZnO}$, and a stoichiometric increase in band gap absorption intensity is observed (Figure 1). Similar results were obtained for the synthesis of $\mathrm{Co}^{2+}$ - and $\mathrm{Ni}^{2+}$-doped $\mathrm{ZnO}$ nanocrystals. ${ }^{32}$

In addition to the $\mathrm{ZnO}$ excitonic absorption, a sub-bandgap absorption feature at $24000 \mathrm{~cm}^{-1}$ is observed that also appears only after 0.45 equiv of base has been added; i.e., its appearance coincides with the appearance of $\mathrm{ZnO}$. This sub-bandgap absorption feature is not observed in pure $\mathrm{ZnO}$ QDs synthesized under these conditions, and it is therefore attributed to the $\mathrm{Mn}^{2+}$. The fact that its appearance accompanies $\mathrm{ZnO}$ formation provides a strong indication that the $\mathrm{Mn}^{2+}$ ions giving rise to this absorption intensity are associated with crystalline $\mathrm{ZnO}$. This intensity is assigned as a charge-transfer transition (see section IV.F for analysis). Because the $\mathrm{Mn}^{2+} \mathrm{d}-\mathrm{d}$ transitions are extremely weak, it was not possible to extract as detailed

(39) LaMer, V. K.; Dinegar, R. H. J. Am. Chem. Soc. 1950, 72, 4847-4854. (40) Tokumoto, M. S.; Pulcinelli, S. H.; Santilli, C. V.; Briois, V. J. Phys. Chem. $\underline{B} \mathbf{2 0 0 3}, 107,568-574$. 
information about the nucleation process from these titration experiments as was possible for $\mathrm{Co}^{2+}: \mathrm{ZnO}$, where the $\mathrm{Co}^{2+}$ ligand-field absorption could be used to follow the reaction. ${ }^{32}$ Instead, EPR spectroscopy was used to probe the $\mathrm{Mn}^{2+}$ ions during synthesis.

The EPR spectra shown in Figure $2 b-d$ were collected at different stages of nanocrystal synthesis under reaction conditions similar to those of Figure 1. They reveal the changing environment of the $\mathrm{Mn}^{2+}$ ions during the preparation. For reference, we have prepared pure $\mathrm{ZnO}$ quantum dots and then deliberately bound $\mathrm{Mn}^{2+}$ ions to their surfaces (i.e., $100 \%$ surface $\mathrm{Mn}^{2+}$ ). The resulting EPR spectrum is shown in Figure $2 \mathrm{a}$. The breadth of the features in Figure $2 \mathrm{a}$ is attributed to the inhomogeneous $\mathrm{Mn}^{2+}$ speciation on the nanocrystal surfaces. The EPR spectrum of $\mathrm{Mn}^{2+}: \mathrm{ZnO}$ nanocrystals collected shortly after addition of base (Figure $2 b$ ) generally resembles that of surface-bound $\mathrm{Mn}^{2+}: \mathrm{ZnO}$ but shows improved resolution of the fine structure. The emergence of structure in this spectrum is attributed to partial incorporation of $\mathrm{Mn}^{2+}$ into the $\mathrm{ZnO}$ lattice. From the energy of the first excitonic absorption feature, the average nanocrystal diameter for this sample is estimated to be ca. $4.0 \mathrm{~nm}$. At this size, even a statistical distribution of the dopants in the $\mathrm{ZnO}$ nanocrystals will result in a high proportion (ca. 25\%) of the $\mathrm{Mn}^{2+}$ ions at the surfaces of the nanocrystals because of the large surface-to-volume ratios. The spectrum in Figure 2c was collected after the nanocrystals were allowed to Ostwald ripen at $60{ }^{\circ} \mathrm{C}$ for $2 \mathrm{~h}$. An average nanocrystal diameter of ca. $5.6 \mathrm{~nm}$ is estimated from the red-shifted excitonic absorption spectrum of these colloids (ca. 20\% surface). The EPR spectrum of Figure 2c is somewhat sharper and shows slightly better resolved hyperfine structure than that of Figure 2b, reflecting increased homogeneity in the $\mathrm{Mn}^{2+}$ speciation. The increased homogeneity thus qualitatively follows the decrease in the surface-to-volume ratio upon increasing the crystal diameters from 4.0 to $5.6 \mathrm{~nm}$. The level of resolved hyperfine structure observed in Figure $2 \mathrm{c}$ is similar to that reported previously for calcined $\mathrm{Mn}^{2+}: \mathrm{ZnO}$ nanocrystals. ${ }^{33}$

B. Removing Dopants from Nanocrystal Surfaces with Dodecylamine. The presence of surface $\mathrm{Mn}^{2+}$ ions lowers the quality of the DMS-QDs and may compromise or obfuscate their physical properties. We have previously demonstrated that $\mathrm{Co}^{2+}$ and $\mathrm{Ni}^{2+}$ ions on $\mathrm{ZnO}$ nanocrystal surfaces can be removed by heating the nanocrystals in technical grade TOPO. ${ }^{32}$ Here we report an improved procedure for removing surface-bound dopants that involves heating the quantum dots in a solution of dodecylamine at $180{ }^{\circ} \mathrm{C}$ for ca. $30 \mathrm{~min}$. The dodecylamine ligates surface-exposed dopants and solvates them, leaving only internal dopants within the cores of the colloidal $\mathrm{ZnO}$ nanocrystals. The $300 \mathrm{~K}$ EPR spectrum of colloidal $\mathrm{Mn}^{2+}: \mathrm{ZnO}$ nanocrystals cleaned in this way (Figure 2d) shows exceptional resolution and rich fine structure. From the excellent agreement between this spectrum and that of bulk $\mathrm{Mn}^{2+}: \mathrm{ZnO}$ (see section IV.C), we conclude that the resulting nanocrystals contain solely internal $\mathrm{Mn}^{2+}$ ions with homogeneous speciation. These data emphasize the importance of removing surface-exposed dopants from DMS-QDs in order to ensure high-quality materials suitable for further study and application.

C. EPR of Surface-Cleaned $\mathrm{Mn}^{2+}: \mathrm{ZnO}$ Nanocrystals. The room-temperature X-and Q-band EPR spectra of the colloidal
$0.02 \% \mathrm{Mn}^{2+}: \mathrm{ZnO}$ nanocrystals (Figure 5) were simulated using the axial spin Hamiltonian given in eq 1.

$$
H=g \mu_{\mathrm{B}} \mathbf{H} \cdot \mathbf{S}+A \mathbf{S} \cdot \mathbf{I}+D\left[S_{z}^{2}-\frac{1}{3} S(S+1)\right]
$$

The first term describes the Zeeman interaction, the second term describes electron-nuclear magnetic hyperfine coupling $\left({ }^{55} \mathrm{Mn}\right.$ nucleus, $I=5 / 2$ ), and the third term accounts for the axial zerofield splitting caused by the hexagonal symmetry of wurtzite $\mathrm{ZnO}$. The $g$ and $A$ tensors were approximated as isotropic in the simulations since the spectral anisotropy is unresolved in the powder-averaged EPR spectrum. ${ }^{41}$ Excellent quantitative agreement with the experimental X- and Q-band spectra could be achieved in simulations (simulations X1 and Q1) using a single set of parameters $\left(g=1.999, A=-74.0 \times 10^{-4} \mathrm{~cm}^{-1}\right.$, and $D=(-2.36 \pm 0.05) \times 10^{-2} \mathrm{~cm}^{-1}$. These parameters agree well with literature values $\left(g_{\|}=1.998, g_{\perp}=2.000, A_{\|}=-74.0\right.$ $\times 10^{-4} \mathrm{~cm}^{-1}, A_{\perp}=-73.5 \times 10^{-4} \mathrm{~cm}^{-1}$, and $D=-2.36 \times$ $10^{-2} \mathrm{~cm}^{-1}$ ) obtained from the study of single crystals of $\mathrm{Mn}^{2+}: \mathrm{ZnO}{ }^{42,43}$ These simulations confirm that the main hyperfine lines in the spectra are due to $\Delta M_{\mathrm{S}}= \pm 1$ transitions having $\Delta M_{\mathrm{I}}=0$ and that the many additional lines arise from the formally forbidden resonances having $\Delta M_{\mathrm{I}} \neq 0$, which gain intensity from off-diagonal terms that are probed in the powderaveraged spectrum. ${ }^{42}$ A small amount of $D$-strain $(\sigma=2 \%$ of $D$ ) was required for optimal simulation of the experimental data (simulations X1 and Q1). For comparison, simulations X2 and Q2 in Figure 5 do not include $D$-strain. Since $D$ originates in the trigonal ligand-field perturbation of the $\mathrm{Mn}^{2+}$ in hexagonal (wurtzite) $\mathrm{ZnO}$, the presence of $D$-strain reflects a greater range of trigonal distortions for the ensemble of $\mathrm{Mn}^{2+}$ ions in the nanocrystals than in bulk $\mathrm{Mn}^{2+}: \mathrm{ZnO}$. This $D$-strain is thus attributable to lattice relaxation effects in the nanocrystals that are not present in bulk $\mathrm{Mn}^{2+}: \mathrm{ZnO}$. In nanocrystalline $\mathrm{Mn}^{2+}: \mathrm{ZnO}, \mathrm{Mn}^{2+}$ ions close to the surface of a nanocrystal will induce greater lattice distortion than those deep in the core because of the crystal's greater capacity to relax structurally near its surface. This $D$-strain is small (2\%), however, and the close match between the simulated and experimental spectra confirms that the $\mathrm{Mn}^{2+}$ ions are substitutionally doped into the $\mathrm{ZnO}$ nanocrystals with very homogeneous speciation. We note that, except for the slight $D$-strain, the ground-state electronic structure of $\mathrm{Mn}^{2+}$ in $\mathrm{ZnO}$ nanocrystals is indistinguishable from that in bulk $\mathrm{ZnO}$ to a high degree of accuracy. This latter point does not support earlier claims that dopant-host electronic interactions may be dramatically enhanced in $\mathrm{Mn}^{2+} \mathrm{DMS}$ nanocrystals and lead to large changes in the electronic structures of the $\mathrm{Mn}^{2+}$ ions, ${ }^{44}$ although the DMS nanocrystals studied here are too large to be strongly quantum confined (section IV.D).

D. Structural Characterization of the $\mathrm{Mn}^{2+}: \mathrm{ZnO}$ Nanocrystals. In addition to solvating surface dopant ions, heating the nanocrystals in dodecylamine also induces further growth by Ostwald ripening. The absorption spectrum of amine-treated $0.20 \% \mathrm{Mn}^{2+}: \mathrm{ZnO}$ nanocrystals in Figure $1 \mathrm{~b}$ shows a band gap

(41) Abragam, A.; Bleaney, B. Electron Paramagnetic Resonance of Transition Ions; Dover Publications: Oxford, 1986.

(42) Hausmann, A.; Huppertz, H. J. Phys. Chem. Solids 1968, 29, 1369-1375

(43) Dorain, P. B. Phys. Rev. 1958, 112, 1058-1060.

(44) (a) Bhargava, R. N.; Gallagher, D.; Hong, X.; Nurmikko, A. Phys. Rev. Lett. 1994, 72, 416-419. (b) Chen, W.; Sammynaiken, R.; Huang, Y.J. Appl. Phys. 2000, 88, 5188-5193. (c) Yan, K.; Duan, C.; Ma, Y.; Xia, S.; Krupa, J.-C. Phys. Rev. B 1998, 58, 13585-13589. 
that is clearly smaller than that of the initially prepared QDs in DMSO shown in Figure 1a. The slight quantum confinement apparent from Figure 1b suggests that the nanocrystal diameters are between 6 and $7 \mathrm{~nm}(\mathrm{ZnO}$ Bohr radius $=3.5 \mathrm{~nm})$, although estimates of $\mathrm{ZnO}$ nanocrystal sizes by band gap measurements are not particularly reliable in this size range. Analysis of the broadened XRD peaks observed for these $0.20 \% \mathrm{Mn}^{2+}: \mathrm{ZnO}$ nanocrystals (Figure 3a) using the Scherrer equation yields an average nanocrystal diameter of $6.1 \mathrm{~nm}$, consistent with the absorption data. Similarly, TEM images of the same nanocrystals (Figure $3 b-d$ ) confirm the crystallinity and pseudo-spherical shapes of these crystals and yield an average crystal diameter of $6.1 \pm 0.7 \mathrm{~nm}$.

E. $\mathrm{Zn}(\mathrm{OAc})_{2}$ Inhibition of $\mathrm{Mn}(\mathrm{OAc})_{2}$ Oxidation in DMSO. $\mathrm{Mn}^{2+}$ is easily oxidized in air to form oxides such as $\mathrm{Mn}_{2} \mathrm{O}_{3}$ and $\mathrm{Mn}_{3} \mathrm{O}_{4}$, and identification of appropriate reducing conditions that allow the preparation of $\mathrm{Mn}^{2+}$-doped $\mathrm{ZnO}$ by hightemperature methods has been extensively investigated. ${ }^{45}$ To maintain $\mathrm{MnO}$ as divalent $\mathrm{Mn}^{2+}$ at $900{ }^{\circ} \mathrm{C}$, for example, $\mathrm{O}_{2}$ pressures below $10^{-7}$ atm are required. Similarly, $\mathrm{Mn}^{2+}$ solutions are sensitive to oxidation in air at room temperature, and stock solutions of $\mathrm{Mn}(\mathrm{OAc})_{2}$ in DMSO stored under air will turn from colorless to brown over a period of several days. Absorption spectra of a solution of $0.002 \mathrm{M} \mathrm{Mn}(\mathrm{OAc})_{2} \cdot 4 \mathrm{H}_{2} \mathrm{O}$ in DMSO collected immediately after preparation and again after 5 days are shown in the inset of Figure 4. The brown color arises from a broad, tailing absorption feature having a shoulder at 20000 $\mathrm{cm}^{-1}$, clearly distinguishable from the substitutional $\mathrm{Mn}^{2+}: \mathrm{ZnO}$ sub-bandgap absorption at $24000 \mathrm{~cm}^{-1}$ (Figure 1). A control experiment in which a similar $\mathrm{Mn}^{2+}$ solution was prepared and stored anaerobically showed $84 \%$ less discoloration after 5 days (Figure 4, $\mathbf{0}$ ), verifying that the discoloration involves oxidation of $\mathrm{Mn}^{2+}$ by atmospheric $\mathrm{O}_{2}$. Basic solutions of $\mathrm{Mn}^{2+}$ are known to convert readily to a variety of complex oxides and hydroxides under air, ${ }^{46}$ such as described by eq 2, and many of these oxidation products are deeply colored.

$$
\mathrm{Mn}^{2+}+2 \mathrm{OH}^{-}+\mathrm{O}_{2} \rightarrow \mathrm{MnO}_{x} \cdot n \mathrm{H}_{2} \mathrm{O}
$$

During our studies of the synthesis of $\mathrm{Mn}^{2+}: \mathrm{ZnO}$ from solution, we observed that similar stock solutions of $\mathrm{Mn}^{2+}$ that also contained $\mathrm{Zn}(\mathrm{OAc})_{2}$ do not change color in air after several days. This observation suggests that $\mathrm{Zn}(\mathrm{OAc})_{2}$ inhibits the oxidation of $\mathrm{Mn}^{2+}$. Data collected for a range of $\mathrm{Zn}(\mathrm{OAc})_{2}$. $2 \mathrm{H}_{2} \mathrm{O}$ concentrations (Figure 4) reveal that inhibition of $\mathrm{Mn}^{2+}$ oxidation is strongly dependent on the concentration of $\mathrm{Zn}$ $(\mathrm{OAc})_{2}$, with almost complete inhibition achieved at $\mathrm{Zn}: \mathrm{Mn}$ stoichiometries as low as only ca. 3:1 (compared to the $\mathrm{Mn}^{2+}: \mathrm{ZnO}$ synthesis conditions of $>49: 1$ used here). In other control experiments, $0.002 \mathrm{M} \mathrm{Mn}\left(\mathrm{NO}_{3}\right)_{2}$ was stored under air for 5 days and showed almost no color change (Figure 4, $\diamond$ ), but addition of $\mathrm{Na}(\mathrm{OAc})$ to a $0.002 \mathrm{M} \mathrm{Mn}(\mathrm{OAc})_{2}$ solution resulted in a substantial increase in discoloration (Figure 4, $\mathbf{\Delta}$ ), suggesting that the acetate actually promotes the oxidation of $\mathrm{Mn}^{2+}$. These data are consistent with the conclusion that the inhibition of $\mathrm{Mn}^{2+}$ oxidation by $\mathrm{Zn}(\mathrm{OAc})_{2}$ is due to $\mathrm{pH}$ effects. Although acetate is a weak base, it is apparently able to

(45) (a) White, W. B.; McIlvried, K. E. Trans. Br. Ceram. Soc. 1965, 64, 521530. (b) Hahn, W. C., Jr.; Muan, A. Am. J. Sci. 1960, 258, 66-78.

(46) Cotton, F. A.; Wilkinson, G. Advanced Inorganic Chemistry, 2nd ed.; Interscience: New York, 1967.
Scheme 1

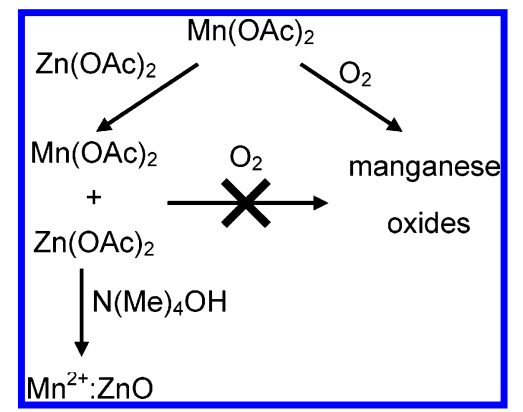

accelerate the oxidation of $\mathrm{Mn}^{2+}$, which should be strongly $\mathrm{pH}$ dependent, as described by eq 2 . Nitrate, an even weaker base, is not as capable of accelerating this reaction. $\mathrm{Zn}^{2+}$ has the opposite effect, decreasing the $\mathrm{pH}$ by lowering the $\mathrm{p} K_{\mathrm{a}}$ of $\mathrm{Zn}^{2+}$ bound water molecules, and thereby inhibiting the $\mathrm{Mn}^{2+}$ oxidation reaction that would otherwise occur. When base is added to this mixture during the synthesis of $\mathrm{Mn}^{2+}: \mathrm{ZnO}$ nanocrystals, we also do not observe the appearance of an absorption shoulder at $20000 \mathrm{~cm}^{-1}$ (Figure 1), and it is reasonable to assume that the small amount of $\mathrm{Mn}^{2+}$ present (in comparison with $\mathrm{Zn}^{2+}$ ) is readily incorporated into basic zinc acetate clusters as they form, thereby prohibiting its coalescence into manganese-rich phases. Incorporation of $\mathrm{Co}^{2+}$ into basic zinc acetate clusters was also concluded from a combination of absorption spectra and nucleation inhibition data. ${ }^{32}$ The conclusion that manganese oxides are not formed is supported by the observation that all of the manganese present in the final product can be accounted for as paramagnetic $S=5 / 2 \mathrm{Mn}^{2+}$ (section IV.H), whereas manganese-rich phases would show exchange-dominated magnetism. In summary, the formation of phase-segregated manganese oxides appears to be prevented very effectively by the high concentration of $\mathrm{Zn}(\mathrm{OAc})_{2}$ also present in the reaction mixture. This inhibition allows high-quality $\mathrm{Mn}^{2+}: \mathrm{ZnO}$ to be synthesized under aerobic conditions without addition of reductants to combat manganese oxidation. These conclusions are summarized in Scheme 1.

F. Charge-Transfer Transitions in $\mathrm{Mn}^{2+}: \mathbf{Z n O}$. The absorption spectra of $\mathrm{Mn}^{2+}: \mathrm{ZnO}$ colloids and thin films show only one feature in addition to the absorption of $\mathrm{ZnO}$, namely a broad tailing sub-bandgap absorbance at ca. $24000 \mathrm{~cm}^{-1}$ (Figures 1 and 6). Although similar broad absorption occurs in many manganese oxides, this feature is unambiguously associated with magnetically dilute $\mathrm{Mn}^{2+}$ ions. This conclusion is drawn on the basis of the MCD spectroscopy of the free-standing $\mathrm{Mn}^{2+}: \mathrm{ZnO}$ nanocrystals shown in Figure 6, in which the broad absorption feature is seen to give rise to a similarly broad negative pseudo- $A$ term MCD signal centered at $23600 \mathrm{~cm}^{-1}$. The $5 \mathrm{~K}$ MCD intensity shows $S=5 / 2$ saturation magnetization (Figure $6)$, demonstrating definitively that this intensity arises from an $S=5 / 2$ chromophore and hence from magnetically isolated $\mathrm{Mn}^{2+}$ ions. The negative MCD signal to higher energy of the pseudo- $\mathcal{A}$ signal is the onset of the first excitonic transition, which gains MCD intensity primarily through the covalency of the $\mathrm{Mn}^{2+}-$ oxo bonds ( $\mathrm{p}-\mathrm{d}$ exchange). ${ }^{1}$ The first excitonic transition is subject to so-called "giant Zeeman splittings", ${ }^{1}$ and the large shift in its energy with applied magnetic field results in nonsuperimposable MCD spectra at different fields in this energy region. Quantitative analysis of this Zeeman shift is currently being investigated and will be reported separately. 
Broad sub-bandgap absorption features similar to the one we observe have been reported previously for other $\mathrm{Mn}^{2+}: \mathrm{ZnO}$ preparations ${ }^{17,22,37,38}$ and have been assigned variously as $\mathrm{Mn}^{2+}$ ligand-field $22,37,38$ or charge-transfer $(\mathrm{CT})^{17}$ transitions. Using the high-quality colloids prepared here, the molar extinction coefficient (per $\mathrm{Mn}^{2+}$ ) of this feature was determined accurately to be $\epsilon_{\mathrm{Mn}^{2+}}=950 \mathrm{M}^{-1} \mathrm{~cm}^{-1}$ at $24000 \mathrm{~cm}^{-1}$ and $300 \mathrm{~K}$. This sub-bandgap absorption feature is approximately 2 orders of magnitude more intense than could be reasonably expected for

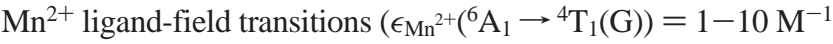
$\mathrm{cm}^{-1}$ in tetrahedral coordination complexes ${ }^{47}$ and in $\mathrm{ZnS}^{48}$ ). Furthermore, the $5 \mathrm{~K} \mathrm{MCD}$ spectrum in this region is structureless (Figure 6), in contrast with what would be observed were this intensity to arise from the closely spaced ${ }^{6} \mathrm{~A}_{1} \rightarrow$ ${ }^{4} \mathrm{~T}_{1}(\mathrm{G}),{ }^{4} \mathrm{~T}_{2}(\mathrm{G}),{ }^{4} \mathrm{~A}_{1}(\mathrm{G}),{ }^{4} \mathrm{E}(\mathrm{G})$ series of $\mathrm{Mn}^{2+}$ ligand-field transitions expected to occur in this same energy region (see Supporting Information). Structured diffuse reflectance spectra in this energy region have been reported ${ }^{22}$ but only at relatively high $\mathrm{Mn}^{2+}$ concentrations where dimer exchange effects may contribute to relaxation of the spin selection rules, and similar structure was not observed in thin films prepared at lower $\mathrm{Mn}^{2+}$ concentrations. ${ }^{17,37,38}$ The intensity and band shape of this subbandgap absorption feature are thus inconsistent with its identification as $\mathrm{Mn}^{2+}$ ligand-field transitions and suggest it should be assigned as CT intensity. We consider two classes of CT transitions that may occur in $\mathrm{Mn}^{2+}: \mathrm{ZnO}$. In one case, an electron may be promoted from the $\mathrm{Mn}^{2+}$ ion to $\mathrm{ZnO}$-based acceptor orbitals of the conduction band (CB), and in the other case an electron may be promoted to the $\mathrm{Mn}^{2+}$ ion from $\mathrm{ZnO}$ based donor orbitals of the valence band (VB). The state generated at the $\mathrm{CT}$ electronic origin is expected to involve a semiconductor electron (or hole) that is loosely bound to the charged impurity by Coulombic forces, ${ }^{49}$ although possibly with a large effective radius. Treating the semiconductor nanocrystal as a ligand to the dopant ion, these transitions are formally analogous to metal-to-ligand CT (MLCT) and ligand-to-metal CT (LMCT) transitions. Increasing the excitation energy may be sufficient to induce photoionization, in which the electron or hole is in an unbound conduction or valence band level. Although binding energies for $\mathrm{Mn}^{2+}$ ions in II-VI semiconductors are not known, typical binding energies for the $\mathrm{VB} \rightarrow \mathrm{TM}^{2+}$ CT excited states in $\mathrm{Ni}^{2+}$-doped II-VI semiconductors are ca. $250 \mathrm{~cm}^{-1} .49$

The intensity of the CT transition observed in the $\mathrm{Mn}^{2+}: \mathrm{ZnO}$ colloids of Figure $1 \mathrm{~b}\left(\epsilon_{\mathrm{Mn}^{2+}} \approx 950 \mathrm{M}^{-1} \mathrm{~cm}^{-1}\right.$ at $\left.24000 \mathrm{~cm}^{-1}\right)$ is comparable to that of the acceptor transition in $\mathrm{Ni}^{2+}: \mathrm{ZnO}$ $\left(\epsilon_{\mathrm{Ni}^{2+}} \approx 700 \mathrm{M}^{-1} \mathrm{~cm}^{-1}\right.$ at $\left.23100 \mathrm{~cm}^{-1}\right),{ }^{32,50}$ suggesting that it may also be a $\mathrm{VB} \rightarrow \mathrm{TM}^{2+} \mathrm{CT}$ transition. Because CT absorption intensities scale with donor-acceptor covalency, ${ }^{51}$ and $\mathrm{s}-\mathrm{d}\left(\mathrm{CB}-\mathrm{Mn}^{2+}\right)$ hybridization is generally an order of magnitude smaller than $\mathrm{p}-\mathrm{d}\left(\mathrm{VB}-\mathrm{Mn}^{2+}\right)$ hybridization in IIVI DMSs, ${ }^{1,7} \mathrm{TM}^{2+} \rightarrow \mathrm{CB}$ CT intensities are expected to be considerably weaker than $\mathrm{VB} \rightarrow \mathrm{TM}^{2+} \mathrm{CT}$ transitions in the

(47) Lever, A. B. P. Inorganic Electronic Spectroscopy, 2nd ed.; Elsevier Science Publishers: Amsterdam, 1984, and references therein.

(48) Dreyhsig, J.; Allen, J. W. J. Phys.: Condens. Matter 1989, 1, 1087-1099.

(49) (a) Noras, J. M.; Allen, J. W. J. Phys. C: Solid State Phys. 1980, 13, 35113521. (b) Heitz, R.; Hoffmann, A.; Broser, I. Phys. Rev. B 1993, 48, 86728682.

(50) Weakliem, H. A. J. Chem. Phys. 1962, 36, 2117-2140.

(51) Solomon, E. I.; Hanson, M. A. In Inorganic Electronic Structure and Spectroscopy; Solomon, E. I., Lever, A. B. P., Eds.; Wiley-Interscience: New York, 1999; Vol. II, pp 1-129. absorption spectra of $\mathrm{Mn}^{2+}: \mathrm{ZnO}$ and other II-VI DMSs. The observed transition occurs much lower in energy than anticipated for a LMCT transition involving $\mathrm{Mn}^{2+}$, however, casting some uncertainty on this assignment.

Charge-transfer transition energies can be analyzed in the context of Jørgensen's optical electronegativity model, ${ }^{52}$ applied previously for analysis of similar broad features observed in the MCD and absorption spectra of $\mathrm{Co}^{2+}: \mathrm{ZnO}$ and $\mathrm{Ni}^{2+}: \mathrm{ZnO}$ DMSs. ${ }^{32}$ Following Jørgensen, eq 3 relates the energy of a CT transition, $E_{\mathrm{CT}}$, to the donor (D) and acceptor (A) electronegativities $(\chi)$, taking into account differences in spin-pairing energies (SPE) between the ground and excited electronic states. ${ }^{47,52}$

$$
E_{\mathrm{CT}}=30000 \mathrm{~cm}^{-1}\left[\chi_{\mathrm{opt}}(\mathrm{D})-\chi_{\mathrm{opt}}(\mathrm{A})\right]+\Delta \mathrm{SPE} \pm 10 D q
$$

The $\triangle$ SPE values for $\mathrm{Mn}^{2+}$ acceptor $\left(\mathrm{d}^{5} \rightarrow \mathrm{d}^{6}\right)$ and donor $\left(\mathrm{d}^{5}\right.$ $\rightarrow \mathrm{d}^{4}$ ) CT transitions are both $+28 / 9[(5 / 2) B+C],{ }^{47}$ where $B$ is the Racah electron-electron repulsion parameter. Reliable ligand-field parameters are not available for $\mathrm{Mn}^{2+}$ in $\mathrm{ZnO}$ and in general are difficult to ascertain for $\mathrm{Mn}^{2+}$ since the energies of the observable ligand-field excited states depend largely on electron-electron repulsion magnitudes. For our analysis, we have estimated $D q=420 \mathrm{~cm}^{-1}, B=596 \mathrm{~cm}^{-1}$, and $C / B=6.5$ by extrapolation of ligand-field parameters determined previously for $\mathrm{Mn}^{2+}, \mathrm{Co}^{2+}$, and $\mathrm{Ni}^{2+}$ tetrahalide complexes ${ }^{47}$ and $\mathrm{Co}^{2+}$ - and $\mathrm{Ni}^{2+}$-doped $\mathrm{ZnO}^{50}$ (see Supporting Information). Although $\mathrm{Mn}^{2+}$ can accept or donate electrons using either the $e$ or $t_{2}$ d-orbitals, correction of eq 3 for the tetrahedral ligandfield splitting $(10 D q)$ is likely necessary because in both cases the CT absorption intensity is dominated by $\mathrm{Mn}^{2+}-\mathrm{ZnO}$ covalency predominantly involving the $t_{2}$ d-orbitals. From absorption spectra of octahedral $\mathrm{MnX}_{2}(\mathrm{X}=\mathrm{Cl}, \mathrm{Br})$, $\chi_{\text {opt }}\left(\mathrm{Mn}^{2+}\left(O_{h}\right)\right)$ has been estimated to be $1.45 .{ }^{53}$ The value of $\chi_{\text {opt }}\left(\mathrm{Mn}^{2+}\left(T_{d}\right)\right)$ is expected to be slightly larger, ${ }^{47}$ but for the current analysis it is sufficient to use the approximate value of 1.45. The optical electronegativities of the valence and conduction bands in pure $\mathrm{ZnO}$ have been estimated previously to be $\chi_{\text {opt }}(\mathrm{CB}) \approx 1.1$ and $\chi_{\mathrm{opt}}(\mathrm{VB}) \approx 2.0 .{ }^{53}$ Using these values, solution of eq 3 yields predicted energies of $E_{\mathrm{LMCT}} \approx 37400$ $\mathrm{cm}^{-1}$ and $E_{\mathrm{MLCT}} \approx 23000 \mathrm{~cm}^{-1}$. Using the relatively well defined electronegativities of tetrahedral $\mathrm{Co}^{2+}$ and $\mathrm{Ni}^{2+}$ ions, $\chi_{\text {opt }}(\mathrm{VB}) \approx 2.4$ was determined from $\mathrm{Ni}^{2+}: \mathrm{ZnO}$ and $\mathrm{Co}^{2+}: \mathrm{ZnO}$ $\mathrm{VB} \rightarrow \mathrm{TM}^{2+} \mathrm{CT}$ transition energies, ${ }^{32}$ and with this value we predict $E_{\mathrm{LMCT}} \approx 49400 \mathrm{~cm}^{-1}$. The high energies predicted for the $\mathrm{VB} \rightarrow \mathrm{Mn}^{2+} \mathrm{CT}$ transition in $\mathrm{Mn}^{2+}: \mathrm{ZnO}$ are consistent with the absence of LMCT transitions in $\mathrm{MnX}_{4}{ }^{2-}(\mathrm{X}=\mathrm{Cl}, \mathrm{Br})$ below $38000 \mathrm{~cm}^{-1} .{ }^{47}$ Because of the high LMCT energies of these analogous tetrahedral complexes and those predicted for $\mathrm{Mn}^{2+}$ in $\mathrm{ZnO}, \mathrm{VB} \rightarrow \mathrm{Mn}^{2+} \mathrm{CT}$ absorption intensity at $24000 \mathrm{~cm}^{-1}$ in $\mathrm{Mn}^{2+}: \mathrm{ZnO}$ appears improbable. Alternatively, the predicted $\mathrm{TM}^{2+} \rightarrow$ CB CT energy $\left(E_{\mathrm{MLCT}} \approx 23000 \mathrm{~cm}^{-1}\right)$ is in reasonable agreement with the experimental energy (ca. $24000 \mathrm{~cm}^{-1}$, Figures 1 and 6), and we tentatively assign this absorption intensity as being due to a $\mathrm{Mn}^{2+} \rightarrow \mathrm{CB}$ excitation. Interestingly, photocurrent action spectra of $\mathrm{Mn}^{2+}: \mathrm{ZnO}$ thin films reveal photocurrents generated with photon energies down to 14500

(52) Jørgensen, C. K. Prog. Inorg. Chem. 1970, 12, 101-158.

(53) Duffy, J. A. J. Chem. Soc., Dalton Trans. 1983, 1475-1478. 
$\mathrm{cm}^{-1} .38$ This activity was attributed to $\mathrm{Mn}^{2+}$ ligand-field absorption but appears to arise from the same transitions observed in the spectra of Figure 6. The photocurrent action begins ca. $10000 \mathrm{~cm}^{-1}$ lower in energy than the first $\mathrm{Mn}^{2+}$ ligand-field excited state $\left({ }^{4} \mathrm{~T}_{1}(\mathrm{G})\right)$, predicted at ca. $24900 \mathrm{~cm}^{-1}$ from the Tanabe-Sugano matrices using the ligand-field parameters listed above (see Supporting Information). The lower energy of the $\mathrm{Mn}^{2+} \rightarrow \mathrm{CB}$ CT excitation as compared to that of the ${ }^{4} \mathrm{~T}_{1}(\mathrm{G}) \mathrm{Mn}^{2+}$ ligand-field excitation has important consequences for the luminescence properties of $\mathrm{Mn}^{2+}: \mathrm{ZnO}$, as described in the following section (section IV.G).

G. Luminescence of $\mathbf{M n}^{2+}: \mathrm{ZnO}$ Nanocrystals. $\mathrm{ZnO}$ nanocrystals are known for their characteristic green luminescence, which originates from $\mathrm{e}^{-}-\mathrm{h}^{+}$recombination involving surface trap states. ${ }^{54}$ Figure 7 shows the $300 \mathrm{~K}$ luminescence spectrum of undoped colloidal $\mathrm{ZnO}$ nanocrystals prepared by the same procedure that was used to make the $\mathrm{Mn}^{2+}$-doped nanocrystals reported here. The green emission, which peaks at about 18600 $\mathrm{cm}^{-1}$, is the same as that reported previously. ${ }^{54}$ In addition, a pronounced UV excitonic emission peak is observed from these colloids. The large ratio of UV:green emission in Figure 7 is not observed in TOPO-capped $\mathrm{ZnO}$ nanocrystals prepared by an analogous procedure ${ }^{32}$ or by hot injection, ${ }^{55}$ and it therefore arises specifically from the use of dodecylamine as the capping ligand, presumably by surface passivation. A similar enhancement of UV emission was reported for $\mathrm{ZnO}$ colloids capped with poly(vinylpyrrolidone) ligands. ${ }^{56}$

$\mathrm{Mn}^{2+}$-doped II-VI semiconductor nanocrystals also commonly show emission from the $\mathrm{Mn}^{2+}{ }^{4} \mathrm{~T}_{1}(\mathrm{G})$ ligand-field excited state. This emission has been observed in $\mathrm{Mn}^{2+}: \mathrm{ZnS},{ }^{25}$ $\mathrm{Mn}^{2+}: \mathrm{ZnSe},{ }^{27}$ and $\mathrm{Mn}^{2+}: \mathrm{CdS}^{26}$ quantum dots at about 17200 $\mathrm{cm}^{-1}(580 \mathrm{~nm})$ with a considerably smaller bandwidth than the surface trap emission. The two $\mathrm{Mn}^{2+}: \mathrm{ZnO}$ nanocrystal samples in Figure 7 show no evidence of $\mathrm{Mn}^{2+}$ emission, however; they show only quenching of the $\mathrm{ZnO}$ excitonic and surface trap emission. The surface trap emission of the pure $\mathrm{ZnO}$ QDs is reduced by ca. $42 \%$ with $0.13 \% \mathrm{Mn}^{2+}$ doping, while the excitonic emission is quenched to a slightly greater extent. At this $\mathrm{Mn}^{2+}$ concentration, each ca. $6.5 \mathrm{~nm}$ diameter nanocrystal contains an average of approximately eight $\mathrm{Mn}^{2+}$ ions (see Supporting Information for statistics). The observation of any $\mathrm{ZnO}$ surface trap emission at all in these doped nanocrystals therefore indicates that $\mathrm{Mn}^{2+}$ is not a particularly effective trap. Almost complete quenching of the surface trap emission is observed with the modest dopant concentration of $1.3 \%$ (Figure 7), however. In no instance was $\mathrm{Mn}^{2+}$ ligand-field emission observed. Quenching of the $\mathrm{ZnO}$ luminescence by $\mathrm{Mn}^{2+}$ and the lack of $\mathrm{Mn}^{2+}$ ligand-field emission in $\mathrm{ZnO}$ have both been noted in previous studies of bulk $\mathrm{Mn}^{2+}: \mathrm{ZnO}^{57}$ These observations are explained by the conclusion drawn in section IV.F

(54) (a) van Dijken, A.; Meulenkamp, E. A.; Vanmaekelbergh, D.; Meijerink, A. J. Phys. Chem. B 2000, 104, 1715-1723. (b) Zhou, H.; Alves, H.; Hofmann, D. M.; Kriegseis, W.; Meyer, B. K.; Kaczmarczyk, G.; Hoffmann, A. Appl. Phys. Lett. 2002, 80, 210-212.

(55) Shim, M.; Guyot-Sionnest, P. J. Am. Chem. Soc. 2001, 123, 11651-11654.

(56) Yang, C. L.; Wang, J. N.; Ge, W. K.; Guo, L.; Yang, S. H.; Shen, D. Z. J. Appl. Phys. 2001, 90, 4489-4493.

(57) (a) Mascher, P.; Liu, M.; Kitai, A. H.; Puff, W. Mater. Sci. Forum 1992 105-110, 1145-1148. (b) The emission recently attributed to the $\mathrm{Mn}^{2+}$ ${ }^{4} \mathrm{~T}_{1} \rightarrow{ }^{6} \mathrm{~A}_{1}$ ligand-field transition in nanocrystalline $\mathrm{Mn}^{2+}: \mathrm{ZnO}$ powders (Bhargava, R. N.; Chhabra, V.; Som, T.; Ekimiv, A.; Taskar, N. Phys. Status Solidi B 2002, 229, 673-680) is indistinguishable from the surface trap emission, and no characterization of the $\mathrm{Mn}^{2+}$ was undertaken to demonstrate doping, so it is likely that the emission was misassigned. that the threshold of the MLCT transition lies below the $\mathrm{Mn}^{2+}$ ${ }^{4} \mathrm{~T}_{1}(\mathrm{G})$ state in $\mathrm{ZnO}$. This low-lying MLCT level would provide a pathway for nonradiative decay of the excited $\mathrm{Mn}^{2+}$ ions. The relatively localized wave functions of the surface trap and $\mathrm{Mn}^{2+}$ electronic states imply that direct quenching of surface trap emission by $\mathrm{Mn}^{2+}$ should proceed by Förster energy transfer, but the extremely low oscillator strengths of the $\mathrm{Mn}^{2+}$ visible absorption transitions indicate that $\mathrm{Mn}^{2+}$ dopants cannot quench surface traps effectively by this mechanism. Rather, it is likely that $\mathrm{Mn}^{2+}$ reduces surface trap emission by competing with surface states in trapping the $\mathrm{ZnO}$ excitonic excitation energy, a conclusion supported by the observation that excitonic emission is quenched somewhat more effectively than surface trap emission in the $0.13 \% \mathrm{Mn}^{2+}: \mathrm{ZnO}$ nanocrystals shown in Figure 7.

H. High- $T_{\mathrm{C}}$ Ferromagnetism in $\mathrm{Mn}^{2+}: \mathrm{ZnO}$ Thin Films. The EPR (Figure 5) and magnetic susceptibility (Figures 8 and 9b) data collected for the free-standing nanocrystals show only the paramagnetic phase of the DMS $\mathrm{Mn}^{2+}: \mathrm{ZnO}$. Neglecting the small zero-field splitting of the $\mathrm{Mn}^{2+}{ }^{6} \mathrm{~A}_{1}$ ground state $(|D|=$ $2.36 \times 10^{-2} \mathrm{~cm}^{-1}$ ), the $5 \mathrm{~K}$ magnetization of free-standing 0.20 $\pm 0.01 \% \mathrm{Mn}^{2+}: \mathrm{ZnO}$ nanocrystals can be reproduced quantitatively using the Brillouin function (eq 4$)^{58}$ and the experimental parameters determined from the EPR simulations discussed in section IV.C ( $S=5 / 2$ and $g=1.999)$, with no fitting.

$$
\begin{array}{r}
M=\frac{1}{2} N g \mu_{\mathrm{B}}\left[(2 S+1) \operatorname{coth}\left((2 S+1)\left(\frac{g \mu_{\mathrm{B}} H}{2 k T}\right)\right)-\right. \\
\left.\operatorname{coth}\left(\frac{g \mu_{\mathrm{B}} H}{2 k T}\right)\right]
\end{array}
$$

Here, $\mu_{\mathrm{B}}$ is the Bohr magneton and $N$ represents the number of $\mathrm{Mn}^{2+}$ ions in the sample, derived from the experimental $\mathrm{Mn}^{2+}$ concentrations determined by ICP-AES. The predicted $5 \mathrm{~K}$ magnetization is superimposed on the experimental data for the free-standing nanocrystals in Figure 9b, and the two are identical within experimental error bars (arising from the error bars in $\mathrm{Mn}^{2+}$ concentration). The quantitative agreement between the experimental and calculated magnetization demonstrates that all of the manganese in these nanocrystals is accounted for as paramagnetic $\mathrm{Mn}^{2+}$ and there is little or no influence from antiferromagnetic superexchange interactions at this low dopant concentration. As mentioned in section IV.E, this result demonstrates that no significant phase segregation of manganese oxides has taken place during synthesis.

When these nanocrystals are spin-coated into thin films, their magnetic properties change dramatically. Films A, B, and C all exhibit robust ferromagnetism at room temperature (Figure $8)$, with relatively minor changes in hysteresis properties over the entire accessible temperature range $(\leq 350 \mathrm{~K}$, Figure S2). The $300 \mathrm{~K}$ saturation moment of $M_{\mathrm{S}}=1.35 \mu_{\mathrm{B}} / \mathrm{Mn}^{2+}$ for film $\mathrm{C}$ exceeds the only other reported $300 \mathrm{~K} \mathrm{Mn}^{2+}: \mathrm{ZnO}$ ferromagnetic saturation moment $\left(M_{\mathrm{S}}=0.16 \mu_{\mathrm{B}} / \mathrm{Mn}^{2+}\right)^{15}$ by nearly an order of magnitude and is similar to the $10 \mathrm{~K} M_{\mathrm{S}}$ value reported recently for a manganese-doped GaAs thin film grown by molecular beam epitaxy and applied in a spin-LED device. ${ }^{9}$

The ferromagnetism probed by magnetic susceptibility (Figures 8 and 9) also manifests itself in the EPR spectroscopy of

(58) Kittel, C. Introduction to Solid State Physics, 7th ed.; Wiley: New York, 1996. 
these thin films (Figure 10). The $300 \mathrm{~K}$ EPR spectrum of the free-standing $0.20 \% \mathrm{Mn}^{2+}: \mathrm{ZnO}$ colloids used to make film A (Figure 10, dashed line) shows the sharp features centered at $g$ $=1.999$ analyzed in Figure 5 for nominally $0.02 \% \mathrm{Mn}^{2+}: \mathrm{ZnO}$ nanocrystals. In contrast, the $300 \mathrm{~K}$ EPR spectrum of film A exhibits a broad resonance spanning the entire field range in Figure 10. This broad feature, a so-called ferromagnetic resonance (FMR) signal, ${ }^{59}$ arises from transitions within the ground state of a ferromagnetic domain. Its breadth likely arises in part from the intrinsic anisotropy of the signal. For example, separations of as large as 5000 Oe are observed between parallel and perpendicular resonance fields in oriented manganese-doped GaAs thin films, ${ }^{59}$ and such large anisotropies could result in broad spectra for powder samples. The breadth may also arise in part from the very high multiplicity of the ferromagnetic domain's ground state due to its high effective spin state (estimated from the $300 \mathrm{~K}$ saturation magnetization curvature in Figure 8 to exceed $S=800$ on average). Finally, inhomogeneity in domain sizes may contribute to the breadth of the signal. A similar $300 \mathrm{~K}$ FMR signal was reported recently for nominally $2 \% \mathrm{Mn}^{2+}: \mathrm{ZnO}$ bulk powders prepared by hightemperature solid-state fusion ${ }^{15}$ and is also observed in ferromagnetic $\mathrm{Co}^{2+}: \mathrm{ZnO}$ thin films prepared by MOCVD. ${ }^{60}$

Using the high-quality colloidal $\mathrm{Mn}^{2+}: \mathrm{ZnO}$ nanocrystals as solution precursors for spin-coating ensures an even distribution of $\mathrm{Mn}^{2+}$ ions throughout the resulting $\mathrm{Mn}^{2+}: \mathrm{ZnO}$ thin film. XRD data collected for film A (Figure 3a) show narrower diffraction peaks than were observed for the paramagnetic nanocrystals. Analysis of the XRD peak widths using the Scherrer equation indicates an increase in effective crystal diameters from 6 to $20 \mathrm{~nm}$, consistent with nanocrystal sintering. There is no evidence of any phase segregation in the XRD data, which show excellent signal-to-noise ratios, although this method would likely be too insensitive to detect phase segregation at such low manganese concentrations. The solid solubility of $\mathrm{Mn}^{2+}$ in $\mathrm{ZnO}$ is high, however, exceeding $10 \%$ at $525^{\circ} \mathrm{C}$ and $1 \mathrm{kbar},{ }^{22}$ and $\mathrm{Mn}^{2+}$ phase segregation during the brief annealing of these $0.20 \% \mathrm{Mn}^{2+}: \mathrm{ZnO}$ nanocrystals at $525^{\circ} \mathrm{C}$ is therefore considered extremely unlikely. ZFC magnetization data have been measured for films A and B, and the two data sets are nearly identical (Figure 9a). Both show increasing magnetization as the temperature is elevated, indicative of spontaneous cooperative magnetization. Notably, no magnetic phase transitions are observed in the ZFC data, confirming the absence of $\mathrm{MnO}$ or $\mathrm{Mn}_{3} \mathrm{O}_{4}$ phase-segregated impurities, nanocrystals of which exhibit magnetic phase transitions below ca. $45 \mathrm{~K}$ that show up as pronounced maxima in ZFC magnetization measurements. ${ }^{61}$

In addition to nearly temperature-independent ferromagnetism, the films show residual paramagnetism that follows Curie behavior in both field and temperature. The paramagnetic contribution in film A was quantified by subtracting the ferromagnetic signal from the $5 \mathrm{~K}$ data in Figure 8 (plotted in

(59) (a) Sasaki, Y.; Liu, X.; Furdyna, J. K.; Palczewska, M.; Szczytko, J.; Twardowski, A. J. Appl. Phys. 2002, 91, 7484-7486. (b) Rubinstein, M.; Hanbicki, A.; Lubitz, P.; Osofsky, M.; Krebs, J. J.; Jonker, B. J. Magn. Magn. Mater. 2002, 250, 164-169.

(60) Schwartz, D. A.; Tuan, A.; Chambers, S. A.; Gamelin, D. R., unpublished results.

(61) (a) Lee, G. H.; Huh, S. H.; Jeong, J. W.; Choi, B. J.; Kim, S. H.; Ri, H.-C J. Am. Chem. Soc. 2002, 124, 12094-12095. (b) Seo, W. S.; Jo, H. H. Lee, K.; Kim, B.; Oh, S. J.; Park, J. T. Angew. Chem., Int. Ed. 2004, 43, $1115-1117$
Figure 9b) and from the $1 \mathrm{~T}$ data at various temperatures (plotted in Figure 9c). Both data sets were then fitted using the Brillouin function (eq 4) with only one floating parameter, $N$, the number of paramagnetic $\mathrm{Mn}^{2+}$ ions. The two data sets agree quantitatively and are best fit when $N=0.64 N_{\text {tot }}$, where $N_{\text {tot }}$ is the total manganese content of the film $(0.20 \pm 0.01 \%)$. The paramagnetic moment per $\mathrm{Mn}^{2+}$ in film A has thus been reduced to ca. $64 \%$ of its value in the free-standing nanocrystals (Figure 9b), and the other $36 \%$ of the $\mathrm{Mn}^{2+}$ ions have undergone a magnetic phase transition. A lower limit for the number of $\mathrm{Mn}^{2+}$ ions participating in the ferromagnetic domains can be estimated from the ferromagnetic saturation moments by assuming a maximum moment of $5 \mu_{\mathrm{B}} / \mathrm{Mn}^{2+}$. With this approximation, $13 \%, 24 \%$, and $27 \%$ of the $\mathrm{Mn}^{2+}$ ions are ferromagnetically aligned at 300 $\mathrm{K}$ for films $\mathrm{A}, \mathrm{B}$, and $\mathrm{C}$, respectively. These numbers represent lower limits, because the apparent moments per $\mathrm{Mn}^{2+}$ will be reduced by the contributions of carriers, antiferromagnetic exchange interactions, or spin-glass behavior. We speculate, for example, that at least some $\mathrm{Mn}^{2+}$ ions are antiferromagnetically exchange-coupled to the ferromagnetic domains as observed previously in $\mathrm{Ni}^{2+}: \mathrm{ZnO} .^{24}$

The EPR signal of paramagnetic $\mathrm{Mn}^{2+}$ in $\mathrm{ZnO}$ can be observed superimposed on the broad FMR signal of film A in Figure 10, consistent with the presence of substantial paramagnetic $\mathrm{Mn}^{2+}$ in these films as concluded from analysis of the magnetic susceptibility data (Figure 9). This paramagnetic EPR signal was not observed in the EPR spectrum of ferromagnetic $\mathrm{Mn}^{2+}: \mathrm{ZnO}$ reported previously, ${ }^{15}$ despite the fact that less than $4 \%$ of the manganese in those samples could be accounted for as ferromagnetic $\left(M_{\mathrm{S}}=0.16 \mu_{\mathrm{B}} / \mathrm{Mn}^{2+}\right)$, leaving more than $96 \%$ of the manganese unaccounted for. The EPR signal of paramagnetic $\mathrm{Mn}^{2+}$ is exceptionally intense at room temperature, and its absence, combined with the weaker ferromagnetism in the material reported previously, may suggest that a substantial portion of the manganese in that material remained antiferromagnetically coupled within $\mathrm{MnO}_{2}$ crystallites, since $\mathrm{MnO}_{2}$ was the original source of manganese used in the synthesis. The stronger ferromagnetism observed in the films prepared from the colloidal doped nanocrystals, coupled with the observation of residual paramagnetic $\mathrm{Mn}^{2+}$, is likely the consequence of a more homogeneous distribution of substitutional $\mathrm{Mn}^{2+}$ dopants throughout the material.

Finally, a new, sharp resonance at $g=2.00(3362 \mathrm{G})$ is observed in the EPR spectrum of the thin film (Figure 10) that was not present in the free-standing colloids. This sharp feature resembles a radical EPR signal and suggests that redox chemistry is occurring during preparation of the thin films. Ferromagnetism in $\mathrm{ZnO}$ DMSs is widely believed to be carrier mediated. ${ }^{2,3}$ Zener model calculations ${ }^{2}$ and local-density-approximation density functional theory (LDA-DFT) calculations ${ }^{3}$ both predict that ferromagnetism in $\mathrm{Mn}^{2+}: \mathrm{ZnO}$ requires the presence of relatively large carrier concentrations $(p \approx 3.5 \times$ $\left.10^{20} \mathrm{~cm}^{-3}\right)$. A sensitive dependence on carrier concentration would be consistent with the observation that $\mathrm{Mn}^{2+}: \mathrm{ZnO}$ prepared by similar methods can show substantially different magnetic properties ${ }^{10,15-18}$ (see also Supporting Information for data on additional films from this study). Importantly, the activating carriers in both models are p-type. In contrast with III-V DMSs such as $\mathrm{Mn}^{2+}$ :GaAs, substitutional doping of $\mathrm{TM}^{2+}$ ions into $\mathrm{ZnO}$ does not itself generate p-type carriers, so 
these carriers must be introduced by other routes. The radicallike feature we observe in the EPR spectrum of the ferromagnetic $\mathrm{Mn}^{2+}: \mathrm{ZnO}$ thin film suggests that redox chemistry during film preparation could possibly be the source of the required carriers. $\mathrm{ZnO}$ forms n-type defects under growth conditions very readily, however, and so it would be surprising if radical chemistry could introduce sufficient p-type defects in our films to explain our magnetic data within the existing theoretical models. Indeed, growth of p-type $\mathrm{ZnO}$ has been a long-standing challenge because of the ubiquity of compensating n-type defects. ${ }^{62}$ Only relatively recently was p-type $\mathrm{ZnO}$ successfully prepared, ${ }^{63}$ by introducing $\mathrm{NO}, \mathrm{NH}_{3}, \mathrm{~N}_{2}$, or $\mathrm{N}_{2} \mathrm{O}$ gases during $\mathrm{ZnO}$ growth to incorporate $\mathrm{N}$ heteroatoms at anion sites of the wurtzite lattice. One intriguing possibility is that calcination of the spin-coated dodecylamine-ligated $\mathrm{Mn}^{2+}: \mathrm{ZnO}$ nanocrystals leaves behind nitrogen heteroatoms that serve as p-type defects by a process analogous to those reported previously. ${ }^{63}$ Further investigation is needed to fully unravel the issues of magnetic ordering mechanism and carrier type in ferromagnetic $\mathrm{Mn}^{2+}: \mathrm{ZnO}$, but the possibility that the chemical identity of the capping ligand may play an important role when starting from nanocrystalline precursors exposes the exciting possibility that room-temperature magnetic ordering in $\mathrm{Mn}^{2+}: \mathrm{ZnO}$ and related DMSs may ultimately be controlled, and hence understood, using chemical perturbations. Experiments exploring these subjects are currently underway.

\section{Conclusion}

We have demonstrated the preparation of high-quality colloidal $\mathrm{Mn}^{2+}$-doped $\mathrm{ZnO}$ diluted magnetic semiconductor quantum dots by a direct solution chemical route. Segregation of dopant phases is a major concern in the synthesis of DMSs by any method because it may interfere with observation of the intrinsic properties of the target DMS. In many ZnO DMS preparations reported previously, methods have been used that may actually promote dopant segregation through high temperatures or reductive conditions, or through difficulties in controlling the form in which dopants are introduced. We have

(62) (a) Zhang, S. B.; Wei, S.-H.; Zunger, A. Phvs. Rev. B 2001, 63, 075205/ 1-7. (b) Look, D. C.; Hemsky, J. W.; Sizelove, J. R. Phvs. Rev. Lett. 1999, 82, 2552-2555.

(63) (a) Li, X.; Yan, Y.; Gessert, T. A.; Perkins, C. L.; Young, D.; DeHart, C. Young, M.; Coutts, T. J. J. Vac. Sci. Technol. A 2003, 21, 1342-1346. (b) Minegishi, K.; Koiwai, Y.; Kikuchi, Y.; Yano, K.; Kasuga, M.; Shimizu, A. Jpn. J. Appl. Phvs. Part 2 (Lett.) 1997, 36, L1453-L1455. (c) Look, D. C.; Reynolds, D. C.; Litton, C. W.; Jones, R. L.; Eason, D. B.; Cantwell, G. Appl. Phvs. Lett. 2002, 81, 1830-1832. (d) Guo, X.-L.; Tabata, H.; Kawai, T. J. Crvst. Growth 2001, 223, 135-139. demonstrated that it is possible to prepare colloidal $\mathrm{Mn}^{2+}: \mathrm{ZnO}$ nanocrystals with extremely homogeneous dopant speciation, in which all of the $\mathrm{Mn}^{2+}$ is substitutionally doped within the cores of the nanocrystals and not on the crystal surfaces, by direct chemical synthesis from homogeneous solution in air at room temperature. Preparation of thin films by spin-coat processing using these colloids as solution precursors yields strongly ferromagnetic $\mathrm{Mn}^{2+}: \mathrm{ZnO}$ thin films with Curie temperatures well above room temperature and with $300 \mathrm{~K}$ saturation moments up to $1.35 \mu_{\mathrm{B}} / \mathrm{Mn}^{2+}$, nearly 1 order of magnitude greater than the only previously reported roomtemperature value for $\mathrm{Mn}^{2+}: \mathrm{ZnO} .{ }^{15}$ These results demonstrate the successful application of direct chemical routes to the preparation of a strongly ferromagnetic semiconductor that has been predicted to play an important role in the emerging field of spin-based electronics technologies. The insights gained from chemical experiments with this and other diluted magnetic semiconductors are expected to help guide the preparation of increasingly high-quality ferromagnetic semiconductors and improve the fundamental understanding of their interesting physical properties.

Acknowledgment. This work was funded by the NSF (DMR0239325 and ECS-0224138). The authors are grateful to the UW/PNNL Joint Institutes for Nanoscience for graduate support (N.S.N., K.R.K.), to Prof. Høgni Weihe (University of Copenhagen) and Prof. Philip Tregenna-Piggott (University of Bern) for invaluable assistance with the EPR simulations, and to Dr. Chongmin Wang (PNNL) and Dr. J. Daniel Bryan (UW) for assistance with TEM measurements. NIH Center grant P30 ES07033 is acknowledged for supporting the X-band EPR instrument at UW. Q-band EPR and TEM measurements were performed at EMSL, a national user facility sponsored by the U.S. DOE's Office of Biological and Environmental Research located at PNNL and operated by Battelle. D.R.G. is a Cottrell Scholar of the Research Corporation.

Supporting Information Available: Three tables compiling literature data used to estimate ligand-field parameters and excited-state energies for $\mathrm{Mn}^{2+}$ in $\mathrm{ZnO}$; three figures showing doping statistics for $\mathrm{Mn}^{2+}: \mathrm{ZnO}$ nanocrystals, temperature dependence of magnetic hysteresis parameters for films $\mathrm{A}-\mathrm{C}$, and $300 \mathrm{~K}$ magnetic data for additional $\mathrm{Mn}^{2+}: \mathrm{ZnO}$ nanocrystalline thin films. This material is available free of charge via the Internet at http://pubs.acs.org.

JA048427J 